\title{
High-Speed PIV of shock boundary layer interactions in the transonic buffet flow of a compressor cascade
}

\author{
Joachim Klinner ${ }^{1}$ (1) $\cdot$ Alexander Hergt ${ }^{2} \cdot$ Sebastian Grund $^{2} \cdot$ Christian E. Willert $^{1}$
}

Received: 5 June 2020 / Revised: 16 December 2020 / Accepted: 11 January 2021

(C) The Author(s) 2021

\begin{abstract}
The dynamics of a transonic buffet flow on the suction side of a highly loaded 2-D compressor blade model is investigated at a chord-based Reynolds number of $1.4 \times 10^{6}$ and an inlet Mach number of 1.05. Near stall the detached bow shock exhibits pronounced modal shock oscillations at buffet frequencies of $\omega^{*}=2 \pi f c / U_{1}=2.27$ which are not related to any structural aeroelastic modes. High-speed PIV at several stations along the chord provides chordwise velocity spectra and wave propagation velocities of shock-induced perturbations. For this purpose, a double-pulse laser system with a high-repetition rate was set up consisting of two combined DPSS lasers. This enables time-resolved PIV using frame straddling at up to $100 \mathrm{kfps}$ and pulse separations down to $800 \mathrm{~ns}$. Synchronized high-speed shadowgraph imaging simultaneously locates the position of the bow shock. Based on cross-correlations between velocity time records at two points and between velocity and shock position, the propagation velocity of the modal perturbations is determined upstream and downstream from the shock. The measured data indicate that feedback occurs between a region immediately downstream of the shock foot and a plane upstream of the shock, up to which transverse velocity disturbances are convected. This observation is contrary to Lee's model Lee (AIAA J 28(5):942-944, 1990) which describes self-sustained shock-buffet as a consequence of shock-trailing edge interactions.
\end{abstract}

\section{Introduction}

In transonic turbomachinery blading, the correct prediction of shock positions and unsteadiness is essential in order to correctly estimate buffet boundary limits as well as frequency, size and location of shock-induced separations. Typically, buffeting is accompanied by severe pressure variations on the blade surface, which in turn increases the structural loading and can shorten machine maintenance cycles, possibly together with an overall reduction in component or machine life. Furthermore, if shock-induced separation occurs in conjunction with buffeting, viscous losses are increased and flow guiding capabilities of the blade passages may reduce. On the other hand, with regard to the prediction of the buffet boundaries for blade design, Giannelis et al.

Joachim Klinner

joachim.klinner@dlr.de

1 German Aerospace Center (DLR), Institute of Propulsion Technology, Engine Measurement Systems, Linder Höhe, D-51147 Köln, Germany

2 German Aerospace Center (DLR), Institute of Propulsion Technology, Fan and Compressor, Linder Höhe, D-51147 Köln, Germany
(2017) in a recent review paper concluded that conclusive explanations for the mechanisms underlying the buffet phenomenon are still lacking. In the literature, there are partly contradictory hypotheses and models about the reasons for self-sustaining shock oscillations that occur without structural excitation.

Lee's model (Lee 1990, 2001) on self-sustained shock oscillations is based on a feedback loop by pressure waves created near the shock foot which convect downstream toward the trailing edge (TE), where they generate upstream propagating waves which induce the shock motion. On this basis and drawing on experiments on a supercritical airfoil, Lee suggested that the buffet period is equal to the sum of the propagation times required for the disturbances to travel from shock to TE and back:

$T_{b}=\int_{\overline{x_{s}}}^{c} 1 / a_{d} \mathrm{~d} x-\int_{c}^{\overline{x_{s}}} 1 / a_{u} \mathrm{~d} x$.

Here $\overline{x_{s}}$ is the mean shock location, $c$ is the chord length, $a_{d}$ is the wave propagation speed in downstream direction (between shock foot and TE) and $a_{u}$ is the upstream wave propagation speed (between TE and shock) and the dominant buffet frequency is $f=1 / T_{b}$. 
Later attempts to verify Lee's model were performed by Hartmann et al. (2013) by measuring wave propagation velocities on a DRA 2303 airfoil on the basis of two-point cross-correlations of time-resolved surface pressure data and time-resolved PIV data along streamlines outside the shockinduced separation region. The authors found experimental evidence for acoustic feedback from the TE, meaning that pressure perturbations which travel over the TE generate upstream traveling sound waves that cause phase-locked variations of the sound pressure level downstream of the shock which displaces the shock. The authors observed considerable overestimation of $f$ for a simplified version of Lee's hypothesis in the form of

$T_{b}=\frac{c-\overline{x_{s}}}{a_{d}}+\frac{c-\overline{x_{s}}}{\left|a_{u}\right|}$,

and argued that acoustic waves propagate circularly from the trailing edge and that shock-motion is induced at the weaker, upper end of the shock. Thus by replacing in the second summand the chordwise distance by the hypotenuse between chordwise distance and shock height resulted in good agreement between experimental data and the predicted buffet frequency.

On the other hand, experimental and numerical studies by Jacquin et al. (2009) and Garnier and Deck (2010) on a supercritical OAT15A profile showed a significant overestimation of the buffet frequency with Eq. (2). The authors argued that other types of feedback must also be considered and found experimental evidence that sound waves generated at the TE can also act on the shock from upstream, after first traveling upstream on the pressure side and then moving around the leading edge onto the suction side.

Feedback in phase with shock motion from the pressure side and from the leading edge was also confirmed numerically by Crouch et al. (2009) who made use of a globalstability theory in combination with unsteady Reynoldsaveraged Navier-Stokes (URANS) simulations to model the buffet phenomenon on a NACA0012 airfoil. Their results are consistent with experimental data but partly in contradiction to Lee's model (Lee 1990), because they also showed that pressure waves generated near the shock foot propagate in the wall normal direction along the shock. When these wall normal waves interact with the lower part of the shock on the suction side, they also spread forward and disperse phaselocked feedback into the oncoming flow.

The investigation presented here was carried out to determine which of the aforementioned feedback mechanisms occur in buffet flows of transonic axial compressor passages operated at compressor relevant conditions and a relatively high angle of attack. A previous study performed by the authors on this type of buffet flow already provided data on the extent of shock-induced separation regions in dependency of the unsteady chordwise shock position (cf. Klinner et al. 2019; Hergt et al. 2019). While this former study was performed using conventional PIV and conditional averaging, the present study utilizes combined high-speed PIV (HS-PIV) and shadowgraph imaging to measure wave propagation velocities of shockinduced perturbations and to identify possible sources of feedback loops that cause self-sustaining shock oscillations in the high-speed cascade flow. Adequate temporal resolution requires sampling rates well above $25 \mathrm{kHz}$, about which very few applications are documented in the current literature for complex configurations such as presented here.

With regard to HS-PIV applications in aerodynamics, Beresh et al. (2015) used a pulse burst laser for large area HS-PIV of a supersonic jet-in-crossflow experiment at $50 \mathrm{kHz}$ sampling, $60 \mathrm{~mJ}$ pulse energy and $10 \mathrm{~ms}$ burst length. Miller et al. (2016) developed a $100 \mathrm{kHz}$ doublepulse burst laser system at pulse energies of $3 \mathrm{~mJ}$ and a burst duration up to $100 \mathrm{~ms}$ which enabled the evaluation of temporal and spatial integral scales in a highly turbulent jet. Recently, Beresh et al. (2018) have extended the application range of their burst laser system to turbulence spectra measurements at $200 \mathrm{kHz}$ sampling (i.e., PostageStamp PIV) corresponding to $400 \mathrm{kHz}$ imaging at pulse energies of $20 \mathrm{~mJ}$.

Instead of using highly complex and expensive pulseburst laser systems, the present investigation makes use of two continuously pulsed industrial grade DPSS lasers with about two orders of magnitude lower pulse energy to generate a well-focused few millimeters wide light-sheet. This allows for HS-PIV in a windtunnel environment at sampling rates of $50 \mathrm{kHz}$, for which the record length is limited only by the available camera memory and beyond which has not been applied in previous investigations of complex transonic aerodynamic configurations.

The present paper is organized as follows: After introducing the operating conditions, the instrumentation and image evaluation schemes for HS-PIV and Shadowgraph imaging are described. The result section starts with a frequency analysis of the shock and blade oscillations followed by results of the turbulence measurements for the inlet flow upstream of the cascade including HS-PIV uncertainty estimates. This is followed by a description of the acquired time-resolved PIV data as well as spatially resolved periodograms and correlations in four regions: upstream of the cascade, upstream of the SBLI, downstream of the SBLI and near the TE. Finally a discussion of the results, including the establishment of a resonance relation based on a characteristic lengths and propagation speeds of perturbations complete the content of the paper. 


\section{Experimental methods}

\subsection{Cascade flow and operation conditions}

The compressor blade cascade (cf. Fig. 1a and Table 1) consists of 6 blades whose geometry is based on a blade cross-section close to mid-span of the first stage rotor of a low pressure compressor of an in-service civil aircraft. The cascade is operated in the transonic cascade wind tunnel of the DLR in Köln (cf. Fig. 1b) which is a closed loop, continuously running facility with a rectangular supersonic nozzle and a variable test section through adjustment of the lower end wall (Hergt et al. 2016). The suction capabilities of the sidewall BLs of the windtunnel were operated at an axial velocity-density ratio near unity, to ensure that shock systems have a low curvature in spanwise direction.

Inlet conditions such as Mach number $\mathrm{Ma}_{1}$, static temperature and speed of sound $c_{1}$ are derived from static pressure measurements at the tunnel sidewalls at the entrance plane MP1 (see Fig. 1) by assuming adiabatic flow and using isentropic gas equations. Two chordwise rows of static pressure taps at mid-span allow monitoring of pressure and Mach number distributions on both pressure and suction side of blade no. 3 .

Flow parameters are adjusted to match specific isentropic Mach-number distributions at mid-span for two operating conditions, either at the aerodynamic design point (ADP) or at an off-design point (ODP, near stall) with respective inflow Mach numbers of 1.12 and 1.05. The off-design point shows pronounced modal shock oscillations and is therefore the subject of the present investigation, while measurements at the ADP represent reference conditions off resonance.

Figure 2 shows schlieren images of the cascade's shock system at the operation conditions that exhibit modal shock oscillations. Due to the relatively low transonic inflow Mach number, the bow shocks in the schlieren
Table 1 Cascade design and flow parameters at off-design conditions (ODP)

\begin{tabular}{lll}
\hline Inlet Mach number & $\mathrm{Ma}_{1}$ & $=1.05$ \\
Reynolds number & $\mathrm{Re}$ & $\approx 1.4 \times 10^{6}$ \\
BL thickness at $x_{c} / c=0.31$ & $\delta_{0}$ & $=0.34 \mathrm{~mm}$ \\
Inlet flow angle & $\beta_{1}$ & $=150.6^{\circ}$ \\
Flow turning angle & $\beta_{1}-\beta_{2}$ & $=9.9^{\circ}$ \\
Stagger angle & $\beta_{s t}$ & $=139.9^{\circ}$ \\
Blade chord length & $\mathrm{c}$ & $=70.0 \mathrm{~mm}$ \\
Pitch & $\mathrm{t}$ & $=49.5 \mathrm{~mm}$ \\
Blade span & $\mathrm{h}$ & $=168 \mathrm{~mm}$ \\
Pitch to chord ratio & $\mathrm{t} / \mathrm{c}$ & $=0.71$ \\
\hline
\end{tabular}

images are straightened and are located a few millimeters upstream from the leading edge and are referred to as detached bow shocks. The Mach number distributions in Fig. 3 confirm that compared to the design point at ODP conditions the bow shock is located on average about 0.05 chord length further upstream.

\subsection{High-Speed PIV instrumentation and data evaluation}

High-speed PIV was conducted at four different stations as indicated in Fig. 1b.

A planar two-component PIV setup is applied using nearly one-to-one imaging and a normal viewing arrangement with the measurement plane located in the central passage of the cascade at mid-span. Tracer illumination was provided with a high-repetition laser system consisting of a pair of diode pumped solid state lasers (Innolas Nanio Air 532-10-V-SP), each providing an average power of up to $10 \mathrm{~W}$. A self-designed beam combination optics allow the individual beams to be superimposed and collimated into a narrow light sheet of $2.5 \mathrm{~mm}$ height and $0.4 \mathrm{~mm}$ thickness (FWHM) in the measurement domain.
Fig. 1 a Definitions of the flow angles and the PIV coordinate system; $\mathbf{b}$ Test section of the cascade windtunnel and measurement stations for HS-PIV:

1. Inflow conditions; 2 . BL upstream of the SBLI; 3. SBLI and downstream BL; 4. TE flow (a)

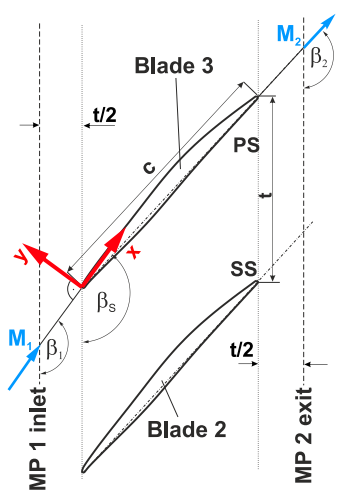

(b)

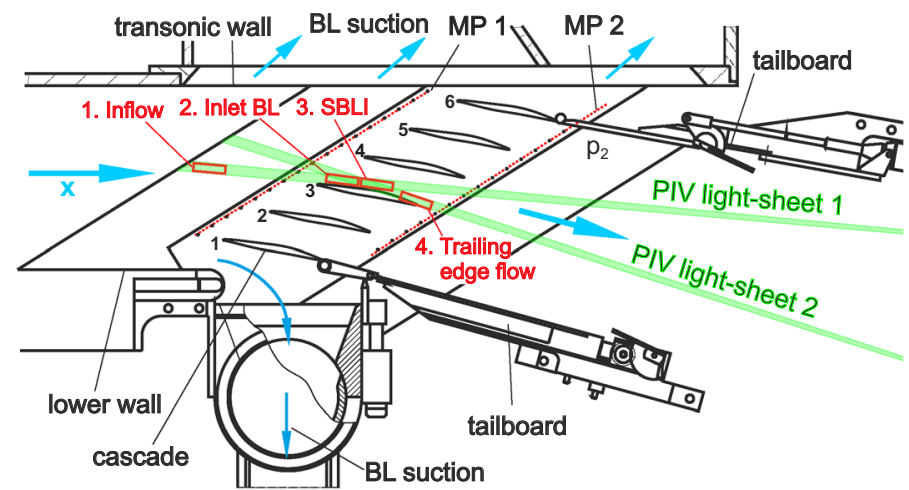


Fig. 2 Schlieren images of the cascade shock system at off-design conditions with pronounced modal shock oscillations (ODP) with measurement regions for high-speed PIV (a)

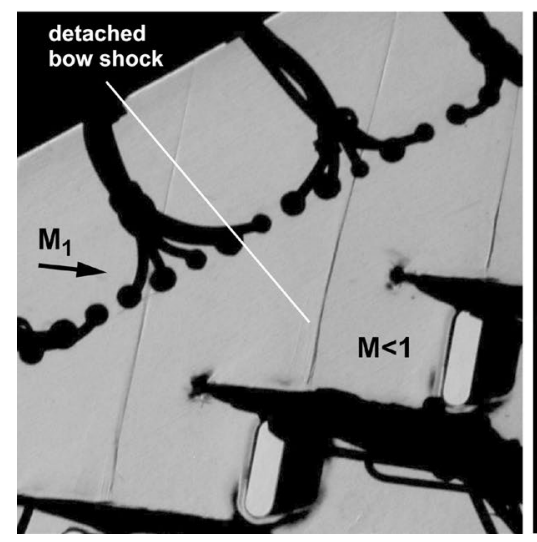

(b)

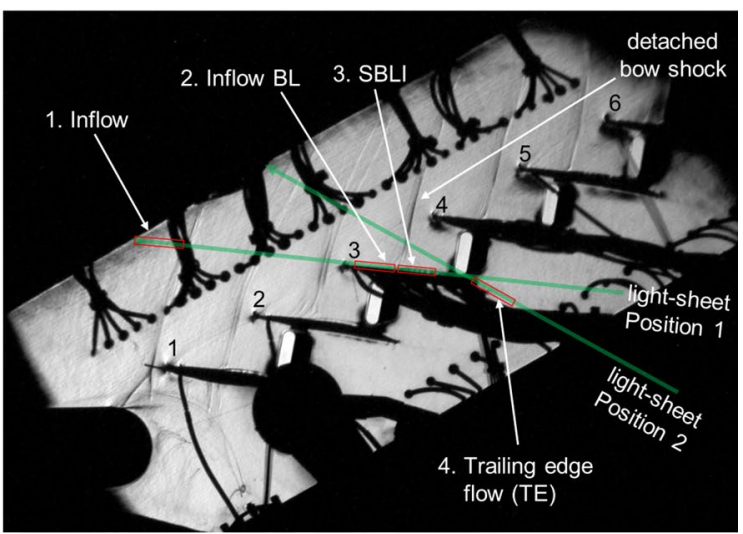

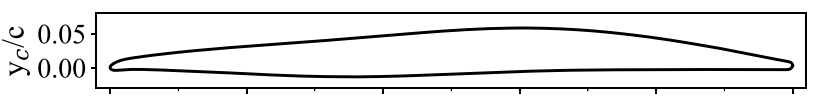

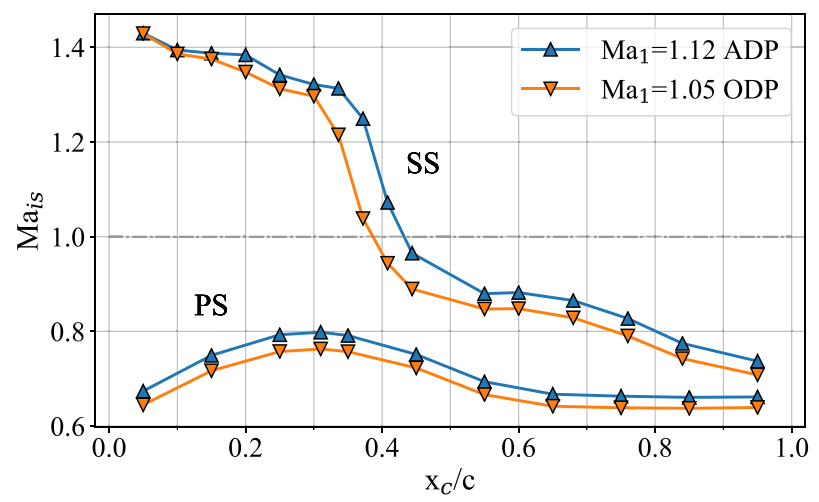

Fig. 3 Isentropic Mach number distributions at mid-span on suction (SS) and pressure (PS) side at the aerodynamic design point (ADP) and at off-design conditions (ODP) where modal shock oscillations occur

The laser beam was delivered to the facility by a mirror arm and a light-sheet probe which is placed 7-8 chord lengths downstream of the cascade trailing edge of blade no. 3 and downstream of the throttle flaps. In this configuration, the laser system provided sufficient energy to illuminate the particles at double pulse repetition rates of $35-50 \mathrm{kHz}$ and typical pulse widths in the order of $20 \mathrm{~ns}$.

The pair of diode pumped lasers used for the high-speed PIV imaging is triggered individually with an external pulse generator. Internal delays control the timing of the Q-switch that ultimately determine the emission of pulsed light by the lasers. This timing not only varies between the lasers but also depends on diode drive currents and other parameters. Therefore the emitted light pulses were monitored by a reverse-biased photo diode with an amplifier of $150 \mathrm{Mhz}$ bandwidth and an oscilloscope to obtain the actual pulse separation along with estimates of pulse jitter. By a correlation-based analysis of the photodiode signal, a constant standard deviation of $6.6 \mathrm{~ns}$ of the pulse separation time $\Delta t$ was found for the whole working range $0.84 \mu \mathrm{s} \leq \Delta t \leq 14 \mu \mathrm{s}$, which corresponds to an uncertainty of $0.8 \%$ for the smallest pulse separation used.

In the present investigation, the cascade wind tunnel is seeded with two different types of seeding. Most PIV measurements are conducted using an atomized paraffin-ethanol mixture of which the ethanol part evaporates upon injection into the tunnel (Klinner et al. 2014). For the characterization of the BL upstream of the shock, a dense smoke oil seeding was used, which produces a significantly larger particle image density in the high-speed flow. The smoke oil based seeding was delivered to the wind tunnel by a smoke generator (Vicount) through a centrifugal pump and a settling chamber in order to separate large particles.

Following the procedures laid out in Klinner et al. (2014), the relaxation length of paraffin oil tracer deceleration to the $1 / \mathrm{e}$ level of the velocity step across a normal shock is $\xi_{p}=0.23 \mathrm{~mm}$ with a corresponding average relaxation time of $\tau_{p}=0.77 \mu \mathrm{s}$. Under a similar shock strength, $\xi_{p}$ has been evaluated with $0.29 \mathrm{~mm}$ for the smoke oil with a corresponding average relaxation time of $\tau_{p}=0.94 \mu \mathrm{s}$.

The double-image recording of the light scattered by the tracers was performed with a Photron Fastcam SA-5 using frame-straddling, meaning that the lasers were fired alternately at the end and at the beginning of the exposures of two consecutive frames. A macro-lens (Nikon Nikkor Micro 200/4) with a magnification set near unity enabled imaging ratios of $21-28 \mu \mathrm{m} / \mathrm{pixel}$ in the measurement volume at a working distance of $270-350 \mathrm{~mm}$. Optical access was through a $5.5 \mathrm{~mm}$ AR-coated glass window and through a 16-mm-thick acrylic glass pane supporting the cascade. Additionally, an anti-peak-locking filter (LaVision) is placed near the image plane to blur particle images over an area covering more than $2 \times 2$ Pixels (Michaelis et al. 2016). After checking the histograms of the PIV subpixel shifts, no evidence of peak-locking was found. 
Table 2 High-speed PIV parameters

\begin{tabular}{lllllll}
\hline Position & $\begin{array}{l}\text { Sample } \\
\text { freq. }[\mathrm{kHz}]\end{array}$ & $\begin{array}{l}\text { Image scale } \\
{[\mu \mathrm{m} / \text { Pixel }]}\end{array}$ & $\begin{array}{l}\text { ROI size W } \\
\times \mathrm{H}\left[\mathrm{mm}^{2}\right]\end{array}$ & $\begin{array}{l}\text { Num. of } \\
\text { records } \times \\
\text { samples }\end{array}$ & $\begin{array}{l}\text { Record } \\
\text { length [s] }\end{array}$ & PIV acc. ${ }^{a}[\mathrm{~m} / \mathrm{s}]$ \\
\hline Inflow & 35 & 22.4 & $21.6 \times 2.3$ & $10 \times 57336$ & 1.63 & $0.4-3.1$ \\
Suction side BL & 42 & 20.5 & $15.8 \times 2.1$ & $2 \times 71671$ & 1.71 & 3.7 \\
SBLI shock foot & 35 & 27.9 & $26.9 \times 2.9$ & $2 \times 57336$ & 1.63 & 3.4 \\
& 50 & 27.9 & $17.9 \times 2.9$ & $2 \times 86005$ & 1.72 & \\
Trailing edge & 35 & 21.0 & $20.2 \times 2.2$ & $2 \times 57336$ & 1.63 & 1.7 \\
& 50 & 21.0 & $13.5 \times 2.2$ & $2 \times 86005$ & 1.72 & \\
\hline
\end{tabular}

${ }^{a}$ based on a positional detection accuracy of the signal peak of 0.15 pixel (cf. Fig. $9 b$ )
Table 2 summarizes sampling frequencies, image field sizes and sequence lengths for high-speed PIV. For each sampling frequency, at least two individual time-sequences were recorded.

The PIV image data were processed using both in-house and commercial software (pyPIView and PIVview v3.8, PIVTEC GmbH) and is based on a coarse-to-fine multi-grid processing scheme with image deformation at each step. To deal with large particle displacements, which arise in order to assess the weak turbulence of the incoming flow, an initial integer offset corresponding to the mean particle image shift was additionally applied. Outliers were excluded based on a normalized median filter (Westerweel and Scarano 2005) and a minimum acceptable correlation coefficient of 0.2 .

The unsteady aerodynamic loading of the thin blade, which is clamped between the two acrylic side walls, results in a flexure of the blade, in particular at mid-span which coincides with the position of the PIV image plane defined by the light sheet. In image space, the range of these vertical blade displacements is \pm 3.5 pixels $( \pm 74 \mu \mathrm{m})$ at maximum for measurements near the trailing edge. Using a correlationbased algorithm, the relative position of the blade surface was determined for each recording in a small rectangular image sample containing the laser flare on the blade suction side. To estimate the image shift in each sample, the intensity distribution in each sample region was correlated with several template images of reference blade positions where each template is shifted by a defined amount in the sub-pixel range. The recovered vertical blade displacements are then used to offset the image data to a coincident blade position with an accuracy of $<0.2$ pixel prior to further PIV processing.

Using a mapping target tailored to match the contour of the blades suction side, the evaluated PIV data are transformed into a uniform coordinate system used throughout this investigation for which $x$ is aligned parallel with the inflow vector at MP1 (cf. Fig. 1a) and the horizontal wind tunnel axis.

From PIV velocity magnitudes, isentropic Mach numbers $\mathrm{Ma}_{\mathrm{is}}$ are obtained by normalization with the speed of sound at the cascade entrance $c_{1}=\sqrt{\kappa R T_{1}}$ where $T_{1}$ is calculated from static pressure measurements at the side walls at MP1 (cf. Fig. 1) using gas equations and assuming an isentropic flow.

\subsection{Shadowgraph setup and evaluation of time-resolved shock positions}

Figure 4 shows the combined setup for visualizing the shock oscillation with shadowgraphs using high-power LEDs (Willert et al. 2010) which flash synchronously with every fourth HS-PIV frame. From earlier work (Klinner et al. 2019), it is known that movements of the shock are well resolved at about $10 \mathrm{kfps}$, which is why the shadowgraph camera records at one fourth the frame rate of the PIV camera. This also has the advantage of capturing a larger field of view that covers the bow shock over two thirds of the passage height.

To optically separate the observation path for shadow images and PIV, a dichroic mirror is used and shadowgraphs were recorded in a different spectral range than PIV using a high-power LED emitting in the red (Luminus Phlatlight CBT90-RX). Equipped with a Nikkor Micro 200/5.6 lens, the shadowgraph camera covers an area of $18.7 \times 14 \mathrm{~mm}^{2}$ at an

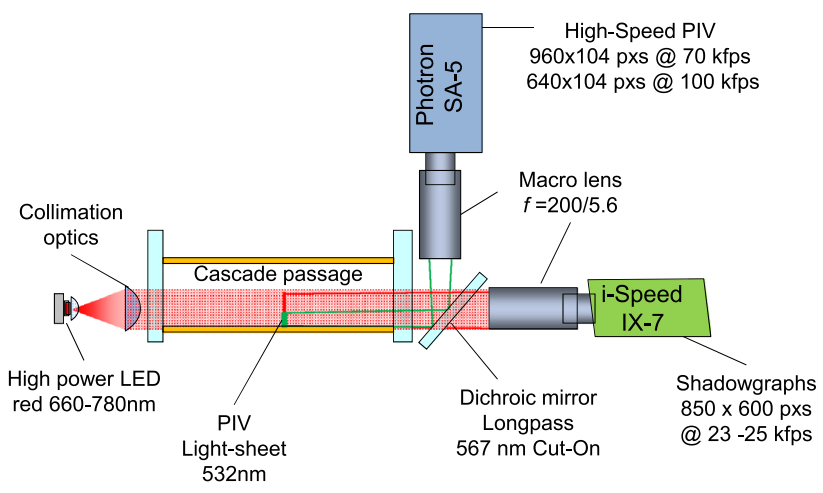

Fig. 4 Combined optical setup for high-speed PIV and shadowgraph visualizations 


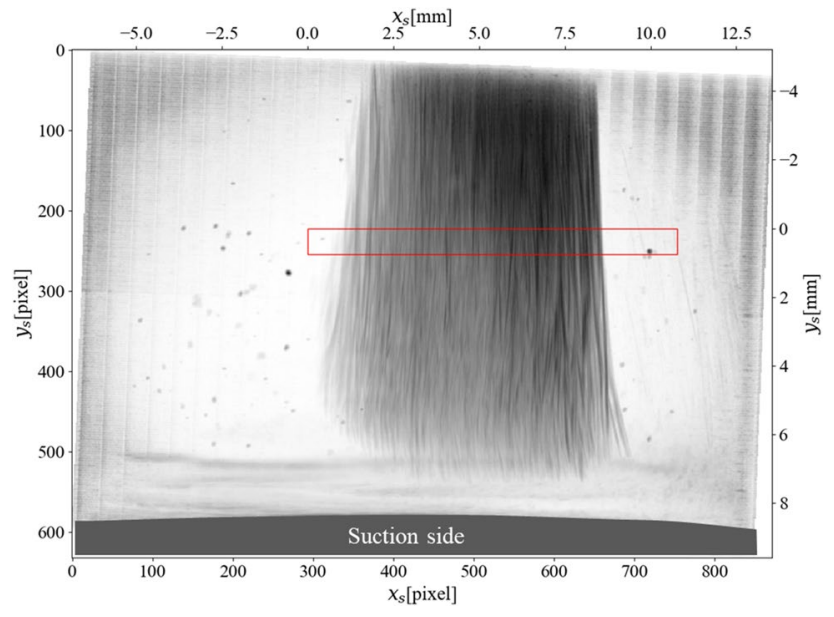

Fig. 5 Minimum image of a shadowgraph sequence visualizing the spatial variation of the detached bow shock near the suction side at the ODP. The red box marks the region in which the shock movement was tracked

image scale of $23 \mu \mathrm{m} / \mathrm{pixel}$. In order to minimize motion blur, the exposure time of shadowgraphs of the oscillating shock is reduced down to $200 \mathrm{~ns}$, corresponding to 0.1 pixel image displacement at the maximum shock motion velocity of $11 \mathrm{~m} / \mathrm{s}$.

Figure 5 shows the intensity minimum over a complete sequence of a duration of $1.7 \mathrm{~s}$ (43,000 shadowgraphs). At the passage shock position, part of the light is deflected more strongly by refractive index gradients, which locally leads to a reduced intensity. Thus, the minimum image shows the fluctuation range of the passage shock position. The red rectangular box marks the area where the shock position is tracked for each single shot. This area is about $8 \mathrm{~mm}$ above the suction side in a region where the bow shock is aligned nearly normal to the flow and thus is undisturbed from unsteady shock splitting near the suction side (i.e., lambda-pattern).

Prior to the automatic detection of the shock position in each single shot, contrast enhancement and flat-field corrections are performed by subtracting each frame from an average image followed by normalization with the average image and image rotation by a certain amount, so that the passage shock in the search area is on average aligned with the image columns. Figure 6 shows a typical timetrace of shock excursions at ODP conditions evaluated in this way. The velocity of the shock motion $u_{s}$ was determined by deriving the temporally resolved shock position using a second-order accurate central difference scheme.
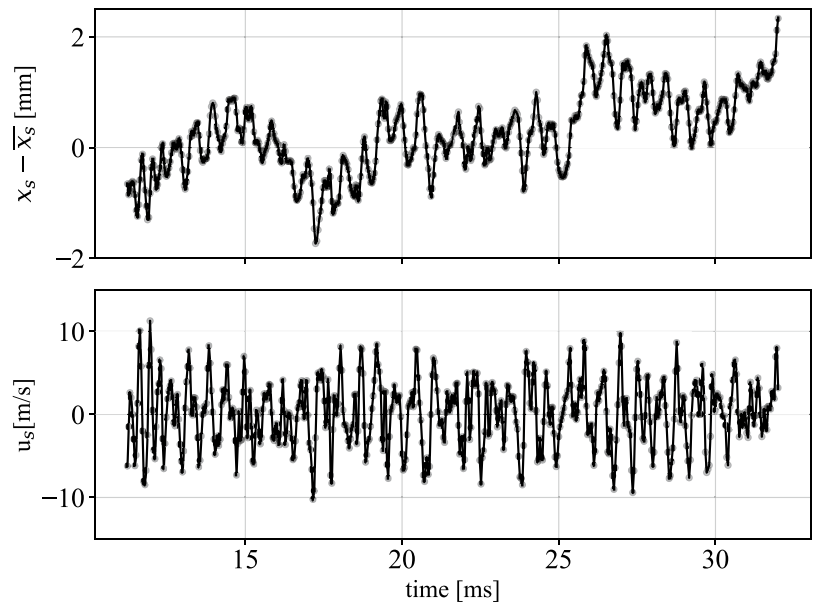

Fig. 6 Example of a time series of the shock excursion at the ODP (top) and corresponding velocity of shock movement (bottom) recorded at $25 \mathrm{kfps}$

\section{Results}

\subsection{Spectral investigation of the buffeting flow}

Figure 7 compares the spectra of the time traces of shock excursions $x_{s}(t)$ retrieved from the time-resolved shadowgraph image sequences. Absolute frequencies $f$ and normalized frequencies $\omega^{*}=2 \pi f c / U_{1}$ are provided for the most dominant spectral peaks. Due to the unsteady spanwise curvature of the three-dimensional shock structure, a detection error between 5 and 10 pixels or 0.1 and $0.2 \mathrm{~mm}$ is assumed as indicated by the beginning roll-up of the spectra near $7 \mathrm{kHz}$. Both spectra show a very similar broad-band shape in the low frequency range up to $1 \mathrm{kHz}$ with peaks at 171 and $210 \mathrm{~Hz}$ and a weak peak around $550 \mathrm{~Hz}$. In the lowfrequency range, broadband components represent the larger shock deflections that contain most of the energy.

In the throttled case (ODP, detached bow-shock) a pronounced frequency-locking of the shock movement occurs at $1.7 \mathrm{kHz}$ and the first harmonic near $3.4 \mathrm{kHz}$ at $\omega^{*}=2.27$ and 4.54. As also indicated in the time trace in Fig. 6, these high-frequency harmonic components modulate low-frequency broad-band shock excursions that extend at maximum over $\pm 4 \%$ of the chord length, indicating that the lock-in behavior at 1.7 and $3.4 \mathrm{kHz}$ does not occur at a fixed distance with respect to the TE. Therefore, the relations 
Fig. 7 Variation of shock buffet depending on operation conditions for two shadowgraph recordings (Seq. 1, 2); a Reference conditions (ADP); b Near stall conditions (ODP) exhibiting a strong lock-in behavior at buffet frequencies above $1 \mathrm{kHz}$; reduced frequen$\operatorname{cies} \omega^{*}=2 \pi f c / U_{1}$ are given in brackets; the bin spacing is $6 \mathrm{~Hz}$

Fig. 8 Wallshift spectra obtained by imaging of the blade surface in two HS-PIV recordings (Seq. 1, 2) at ADP at $\mathrm{Ma}_{1}=1.12 \mathrm{a}$ and at ODP at $\mathrm{Ma}_{1}$ $=1.05$, mainly showing structural modes of blade vibrations; high-frequency noise between 0.1 and 0.2 pixels is suppressed by a low-pass filter; faded colors indicate raw, unfiltered spectral data (a)

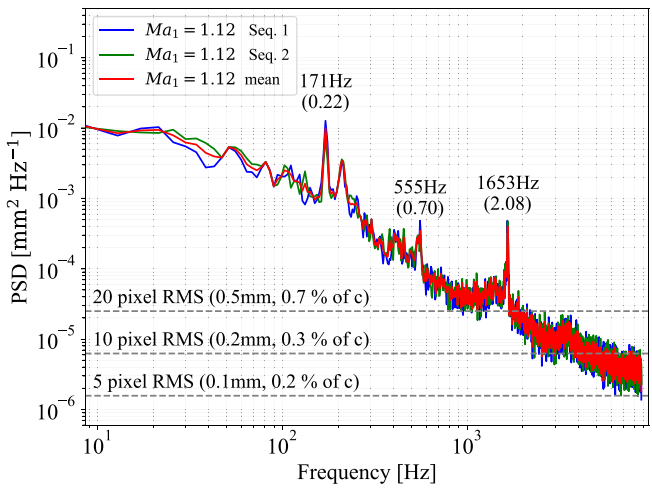

(a)

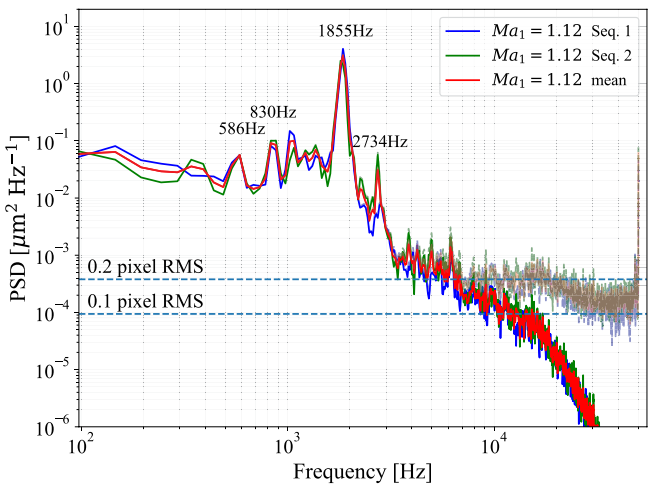

(b)

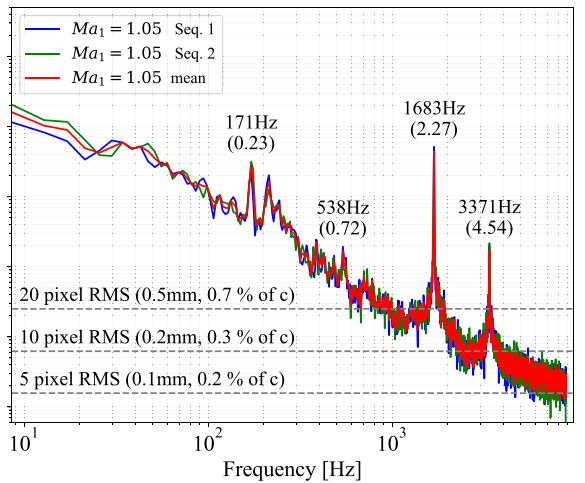

(b)

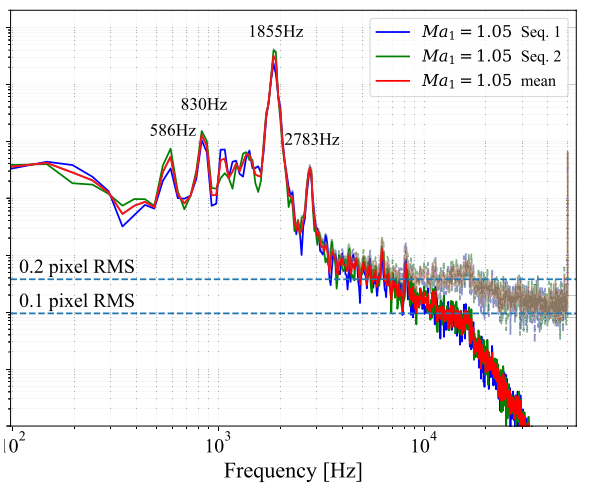

proposed by Lee (2001) (Eq. (1)) and Hartmann et al. (2013) (Eq. (2)) for the evaluation of the buffet frequency on the basis of a fixed distance between shock foot and TE seem not to be applicable for shock oscillations at higher frequencies.

On the other hand, feedback from aeroelastic excitation can be excluded for the following reasons: In contrast to shock-motion spectra, the spectra of the blade deflections in Fig. 8 show hardly any changes between the strongly buffeting flow and the reference case. Local peaks in both spectra correspond to individual structural modes of blade or camera vibrations and the dominant mode at $\approx 1.8 \mathrm{kHz}$ does not coincide with the dominant mode of the shock oscillation. The RMS of the amplitudes of vertical blade displacements is as low as $23 \mu \mathrm{m}$ which is well below $10 \%$ of the boundary BL thickness upstream of the SBLI, which will be determined in Sect. 3.3.

\subsection{Turbulence measurements upstream of the cascade}

Flow conditions upstream of the cascade shock system, such as the turbulence intensity level and the velocity spectrum, are measured in region no. 1 Inflow (see Figs. $1 \mathrm{~b}$ and $2 \mathrm{~b}$ ), which is located near the edge of the schlieren window and is on the edge of just about optically accessible. To distinguish between turbulent fluctuations of the supersonic flow and the PIV noise components, a proposed approach following Scharnowski et al. (2018) was adopted which makes it possible to determine a lower bound of measurement uncertainty of the HS-PIV system, which is strictly taken only valid in the absence of flowdependent error sources as strong local velocity gradients.

From earlier subsonic hot-wire (HW) measurements, it is presumed that the flow at the test section entry exhibits very low turbulence levels in the order of $\sigma_{U} / U=0.5 \%$ where $\sigma_{U}$ denotes the standard deviation of the longitudinal velocity. Assuming that at low turbulence the loss-of-pair error is negligible and that each of the measurement uncertainties is uncorrelated, standard error propagation can be applied as follows:

$\sigma_{\Delta x}^{2}=\left[\frac{\partial \Delta x}{\partial u}\right]^{2} \sigma_{u}^{2}+\left[\frac{\partial \Delta x}{\partial \Delta t}\right]^{2} \sigma_{\Delta t}^{2}+\sigma_{\mathrm{PD}}^{2}$

where $\sigma_{\Delta x}$ is the standard deviations of the measured axial image displacement and $\sigma_{u}$ and $\sigma_{\Delta t}$ are the standard deviations of the axial velocity and of the laser pulse separation time. The standard deviation $\sigma_{\mathrm{PD}}$ represents the random error of the sub-pixel signal peak detection, which depends on imaging quality, the number of particle images per interrogation window and the applied peak fit algorithm. In general, $\Delta x=u \Delta t M$ applies to axial particle image displacements 
between two exposures, with $u$ being an estimate of the local axial velocity, $\Delta t$ being the pulse separation time and $M$ being the magnification. Thus, fluctuating components of axial and transverse displacements are summed up as follows:

$$
\begin{gathered}
\sigma_{\Delta x}^{2}=\frac{\sigma_{u}^{2}}{U^{2}} \Delta x^{2}+\underbrace{M^{2} U^{2} \sigma_{\Delta t}^{2}+\sigma_{\mathrm{PD}}^{2}}_{\sigma_{\mathrm{PV} \Delta x}^{2}} \\
\sigma_{\Delta y}^{2}=\frac{\sigma_{v}^{2}}{U^{2}} \Delta x^{2}+\underbrace{M^{2} V^{2} \sigma_{\Delta t}^{2}+\sigma_{\mathrm{PD}}^{2}}_{\sigma_{\mathrm{PV} \Delta y}^{2}} .
\end{gathered}
$$

Based on Eq. (4), turbulence intensities $\left(\sigma_{u} / U, \sigma_{v} / U\right)$ and PIV uncertainties $\left(\sigma_{\mathrm{PIV} \Delta x}, \sigma_{\mathrm{PIV} \Delta y}\right)$ can be estimated by a quadratic fit based on measurement sequences recorded at several pulse separation times (i.e., several mean particle image shifts).

Figure 9a shows the mean inflow velocities and fluctuations for the inflow region no. 1 normalized by the velocity $u_{1}$ corresponding to the inlet Mach number. Due to access restrictions, the light sheet could not be aligned exactly coinciding with the channel axis, as this would have resulted in shading caused by the cascade. Thus only the lower rows of the ROI could be reliably evaluated, without any in-plane loss-of-pairs. To illustrate this, the maximum applied particle image displacement is marked with the white arrows in Fig. 9a.

Figure $9 \mathrm{~b}$ shows the standard deviation (rms) of the particle image displacements at the sampling positions no. 1 and no. 2 as marked by circles in Fig. 9a for mean particle image displacements between $\Delta x=13-147$ pixels or $0.3-3.3 \mathrm{~mm}$. Based on Eq. (4), the fit parameters regarding turbulence level and PIV uncertainty are shown in the plot legend. It should be noted that due to mean $(v)=0$, the fluctuations of the laser pulse separation $\sigma_{\Delta t}$ primarily influences $\sigma_{\Delta x}$ (see
Eq. (4)) by an uncertainty of 0.09 pixel as represented by the black vertical arrows in Fig. 9b.

The fitted turbulence intensities in Fig. 9b confirm very low levels below $0.5 \%$ as measured with HW. The transverse turbulence intensity is consistently larger than the axial component which is due to the planar supersonic nozzle upstream of the test section which exhibits a contraction along $y$ leading to an increase in the transverse fluctuating velocity components, while stream-wise fluctuating components decrease (cf. Brown et al. 2006). Only marginal influences on the turbulence levels due to PIV sampling window size can be observed.

Integration of the power spectra of the inlet flow in Fig. 10 yields similar turbulence intensity levels compared to the method proposed by Scharnowski et al. (2018), namely $\sigma_{u} / U=0.31 \%$ and $\sigma_{v} / U=0.46 \%$ compared to values at

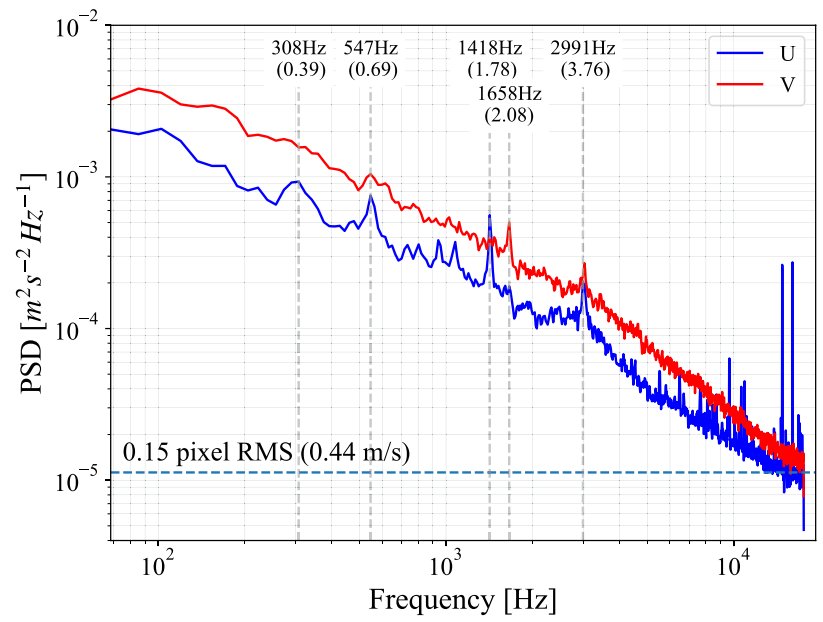

Fig. 10 Velocity spectra at the ADP immediately upstream of the cascade shock system at position no. 1 in Fig. 9a; reduced frequencies in brackets; the bin spacing is $17 \mathrm{~Hz}$
Fig. 9 Quantification of turbulence intensity levels and of the measurement uncertainties for the inflow in region no. 1 in Fig. $2 b$ (a)
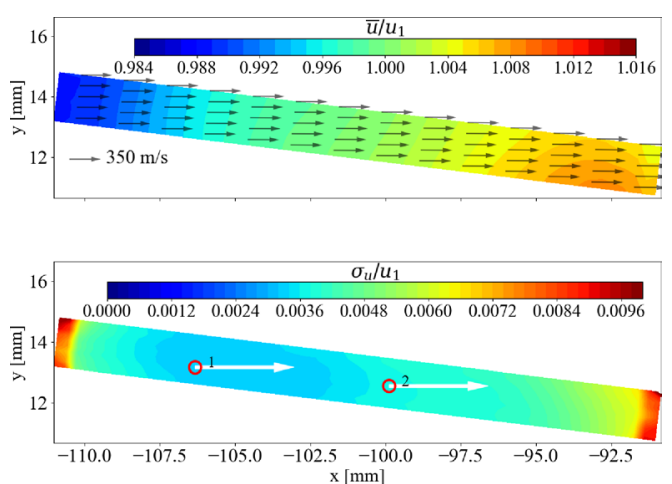

(b)

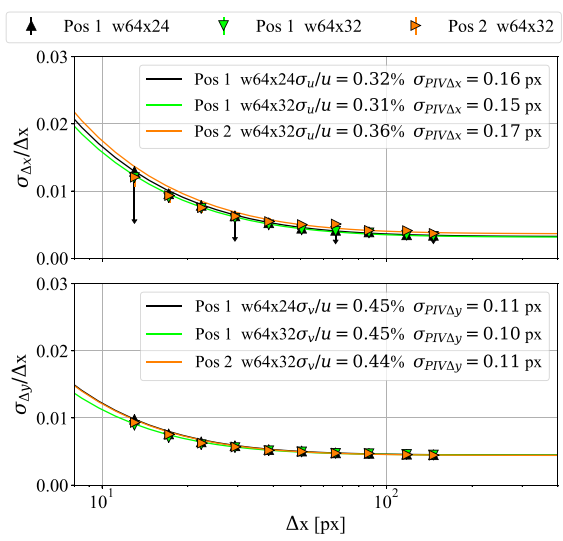


position no. 1 indicated in Fig. $9 \mathrm{~b}$ with $\sigma_{u} / U=0.31-0.32 \%$ and $\sigma_{v} / U=0.45 \%$.

The weak local peaks in the power spectra in Fig. 10 at $f=0.55$ and $1.65 \mathrm{kHz}$ are also observed in the shock motion spectrum at the ADP in Fig. 7a and are caused by the fluctuating detached bow shock near blade tip no. 1 (cf. Fig. 2b), which above already influences the flow in the inlet measuring field No. 1.

\subsection{Boundary layer measurements upstream of the SBLI region}

For the buffeting flow at the ODP, Fig. 11 provides the velocities near the LE obtained with standard 2-c PIV and with 2-c HS-PIV in the region Inflow BL in Fig. 2b. Downstream of the bow-shock, a large subsonic region extends up to the leading edge. The flow is then accelerated to $\mathrm{Ma}>1$ in a Prandtl-Meyer expansion fan and develops a BL. This area is investigated in detail with HS-PIV.

Figure 12a shows enlarged views of a single recording of particle images and the RMS over a HS-PIV image sequence, with the long side of the image parallel to the suction side surface in between column nos. 1 and 2 . The growth of the laminar BL, which is practically devoid of particles, is clearly visible. This particle deficit is due to the strong streamline curvature at the leading edge causing strong wall normal gradients and strong lift forces that act on the particles which, if above a certain inertia threshold, do not remain in the laminar BL. In addition, the closed stream surface of the laminar separation bubble essentially prevents particles from entering this volume. The low RMS intensities in the BL indicate the absence of fluid exchange with the outer flow at this chord-wise location and thus demonstrate the laminar character of the BL.
Fig. 11 Mean isentropic Mach numbers $\mathrm{Ma}_{\mathrm{is}}$ of the leading edge flow measured with standard 2c-PIV and with HS-PIV for the buffeting case (ODP); the HS-PIV region is indicated by the box

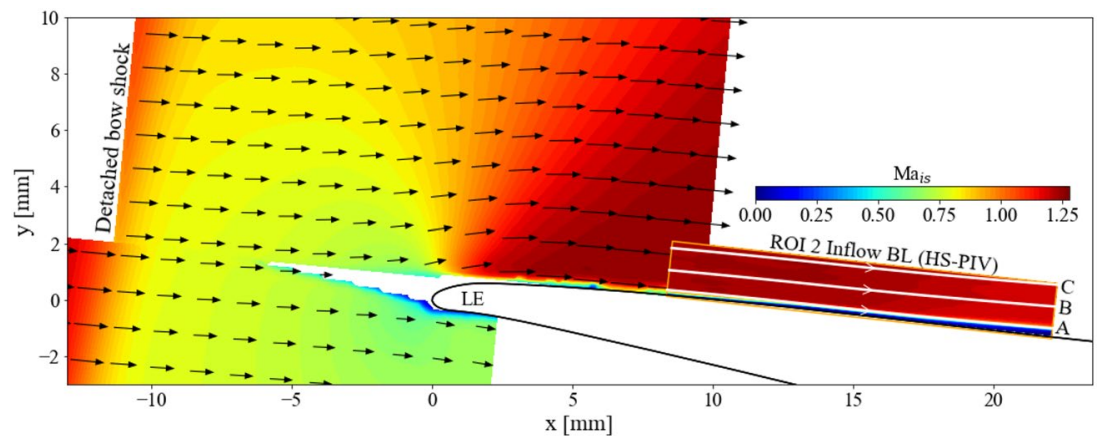

(a)

Fig. 12 a Particle images with dense Vicount seeding taken in the straight section of the suction side surface (indicated by the red line) showing the growth of the laminar BL and the deficit of particles near the blade surface; b Velocity profiles and least-squares-fits to a Blasius BL at columns 1 and 2; the dashed red lines in a represent the BL-thickness from the Blasius fit at columns 1 and 2

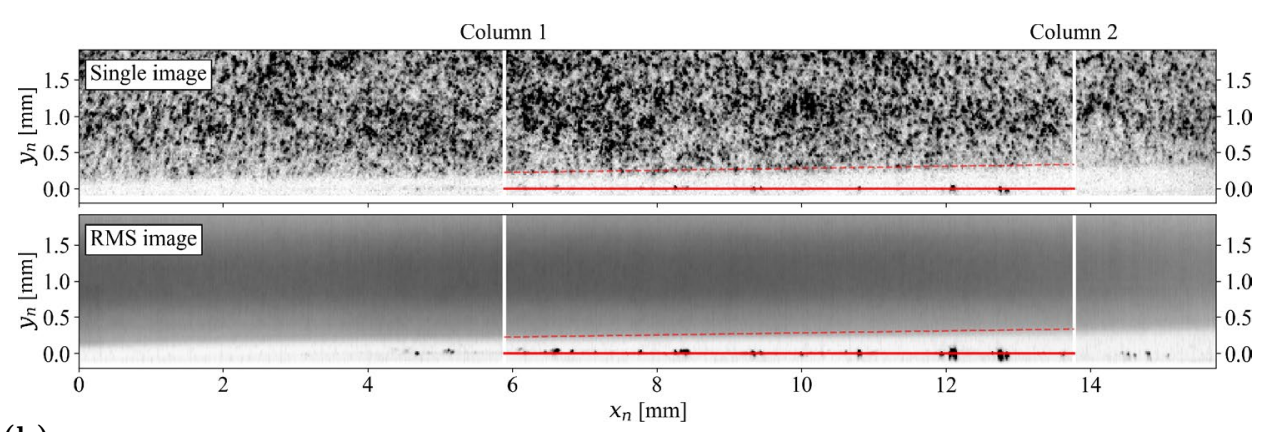

(b)
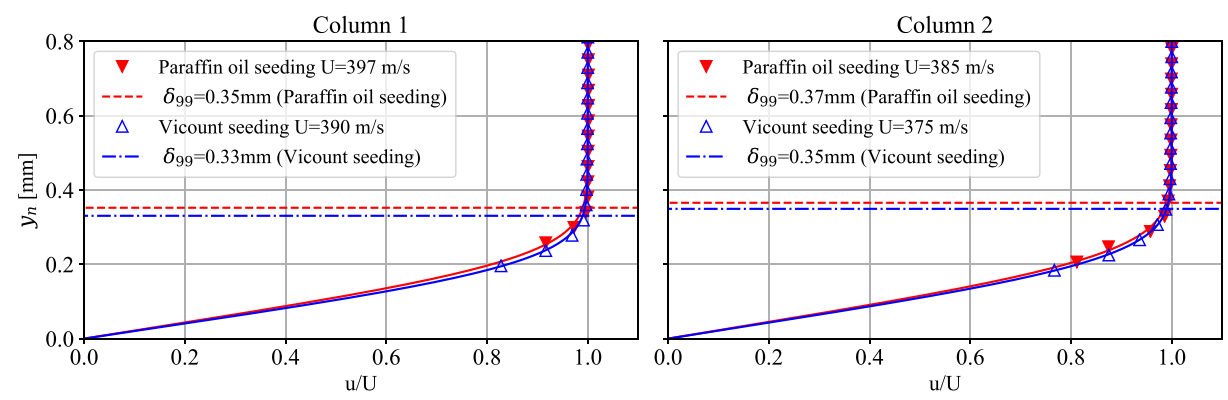
Figure $12 \mathrm{~b}$ shows measured mean axial velocities and the corresponding fits to the Blasius profile for both particle variants at two streamwise locations. As obtained by the Blasius fit, the BL thicknesses $\delta_{99}$ as indicated in the legends of Fig. 12b essentially do not depend on the choice of seeding, that is, smoke oil or paraffin-based seeding. One explanation for this is that despite different mean particle relaxation times of both seeding variants, only particles above a certain inertia remain in the $\mathrm{BL}$ and contribute to the PIV signal at the BL edge. As shown by the dashed line in Fig. $12 \mathrm{a}, \delta_{99}$ lies slightly above the region, where hardly any particles are visible in the BL. Although BL thicknesses are almost identical for both seeding variants, the mean outer velocity $U$ measured with the smoke oil indicates a $2 \%$ lag compared to the velocity measured with the paraffin based seeding. This points to a different capability of both seeding variants to follow the strongly accelerating flow around the leading edge of the suction side and through the expansion fan (cf. Fig. 11).

The BL parameters in Table 3 are obtained from the Blasius fits at both chordwise positions in Fig. 12. Displacement $\delta^{*}$ and momentum $\theta$ thicknesses are estimated by numerical integration of the Blasius fit while neglecting density variations. Under the assumptions that no heat transfer occurs between wall and fluid, density variations are in the order of $30 \%$ based on the ratio of adiabatic wall temperature and static temperatures at both positions. The latter was estimated from total temperature and Mach-number distribution in Fig. 3 assuming isentropic flow. To determine how significant the influence of density variation is on the integral BL scales, the temperature BL was estimated from velocity profiles using the Crocco-Busemann relation, which is valid for a laminar BL over a flat plate in a compressible flow (Schlichting and Gersten 2017). The corresponding densityweighted displacement and momentum thicknesses are $\delta_{c}^{*}$ and $\theta_{c}$. While boundary layer thickness $\delta_{99}$ and displacement thicknesses are slightly lower for the buffeting flow in comparison to the reference, the momentum thickness remains very similar for both operating conditions.

It should be noted that BL thicknesses in Table 3 represent an upper limit and not necessarily correspond to the exact value, since the strong streamline curvature near the leading edge results in an inertia-based selection of the particles, which may bias the measured BL profile. This is also reflected in the relatively high Stokes number of 0.9 for smoke oil and of 0.7 for paraffin oil tracer when related to a BL thickness of $0.4 \mathrm{~mm}$ and based on relaxation times provided in Sect. 2.2.

Although there are only slight variations in the mean BL parameters between the reference and the buffeting flow, for the latter the instantaneous velocities, measured $6 \mathrm{~mm}$ upstream of the shock (column no. 2 in Fig. 12a), already indicate phase-locked modulation with shock motion. To demonstrate this, Fig. 13a shows a timetrace of wall-parallel and transverse velocities $u_{n}$ and $v_{n}$ over a duration of $10 \mathrm{~ms}$. Just above $\delta_{99}$ the wall-parallel velocity $u_{n}$ indicates qualitatively a pronounced modulation from subsonic to transonic around $\mathrm{Ma}=1$ (color shift white to green in the contours). The modulation strength varies with time, that is, the amplitude of the modulation is weaker from 182 to $184 \mathrm{~ms}$, which is probably due to the varying distance to the moving shock foot. Furthermore, variations of the transverse velocity are visible over the entire height of the light sheet. The indicated temporal window of about $8 \times 0.32 \mathrm{~ms}$ corresponds to eight cycles with an average frequency of $3.2 \mathrm{kHz}$ which is close to the first harmonic buffet oscillation $\left(3.37 \mathrm{kHz}, \omega^{*}=4.5\right)$.

Peak-to-peak variations of the amplitudes of $v_{n}$ are in the order of $4 \%$ of $u_{n}$ and are accompanied with a temporally varying flow angle $\beta=\operatorname{atan}\left(v_{n} / u_{n}\right)$ between 0 and $4^{\circ}$ over the entire light-sheet height (cf. Fig. 13b). Variations of velocity magnitude in the same temporal window are in the order of $1 \%$ and do not show a clear modulation. It should be noted that the observed modulation of the velocity near $3.2 \mathrm{kHz}$ is not related to the blade's vibration, shown in the lowest subplot of Fig. 13b and accounted for by a-priori image processing (see Sect. 2.2).

This is also evident from cross-correlations between wallshifts and the wall-normal velocity which revealed a weak periodic correlation at correlation coefficients between $-0.1<R_{i j}<0.2$ but at frequencies between $365 \mathrm{~Hz}$ and $372 \mathrm{~Hz}$ also visible in the PSDs in Fig. 14a. This low-frequency blade or camera oscillation mode is thus in a frequency range lower by a factor of 5-10 than the dominant shock buffet frequencies. For a complete imaging sequence, the range of these vertical blade displacements is \pm 3.3 pixels $( \pm 69 \mu \mathrm{m})$ at maximum, corresponding to one fifth of the BL thickness $\delta_{0}$ at $x_{c} / c=0.31$.
Table 3 Estimates of BL parameters obtained from Blasius fits (cf. Fig. 12) for reference conditions ADP and for the buffeting flow ODP; $\delta_{c}^{*}$ and $\theta_{c}$ include densityweighting using the CroccoBusemann relation

\begin{tabular}{llllllll}
\hline & $x_{c} / c$ & $\delta_{99}$ & $\delta^{*}$ & $\delta_{c}^{*}$ & $\theta$ & $\theta_{c}$ & $\mathrm{H}$ \\
{$[-]$} & {$[\mu \mathrm{m}]$} & {$[\mu \mathrm{m}]$} & {$[\mu \mathrm{m}]$} & {$[\mu \mathrm{m}]$} & {$[\mu \mathrm{m}]$} & $\delta^{*} / \theta$ \\
\hline ADP & $0.19($ Col. 1$)$ & 331 & 116 & 135 & 45 & 38 & 2.58 \\
ODP & $0.19($ Col. 1$)$ & 328 & 115 & 133 & 44 & 38 & 2.59 \\
ADP & $0.31($ Col. 2) & 349 & 122 & 141 & 47 & 40 & 2.59 \\
ODP & $0.31($ Col. 2) & $339=\delta_{0}$ & 119 & 136 & 46 & 39 & 2.59 \\
\hline
\end{tabular}


Fig. 13 a Time-resolved wallparallel and transverse velocities in the inflow BL within the wall-normal column no. 2 (see Fig. 12) located $6 \mathrm{~mm}$ upstream of mean shock foot location; $\mathbf{b}$ time-resolved variation of the inflow angle $\beta=\operatorname{atan}\left(v_{n} / u_{n}\right)$ and magnitude in row $R 1$, marked by a dashed line in a as well as the corresponding wall shift

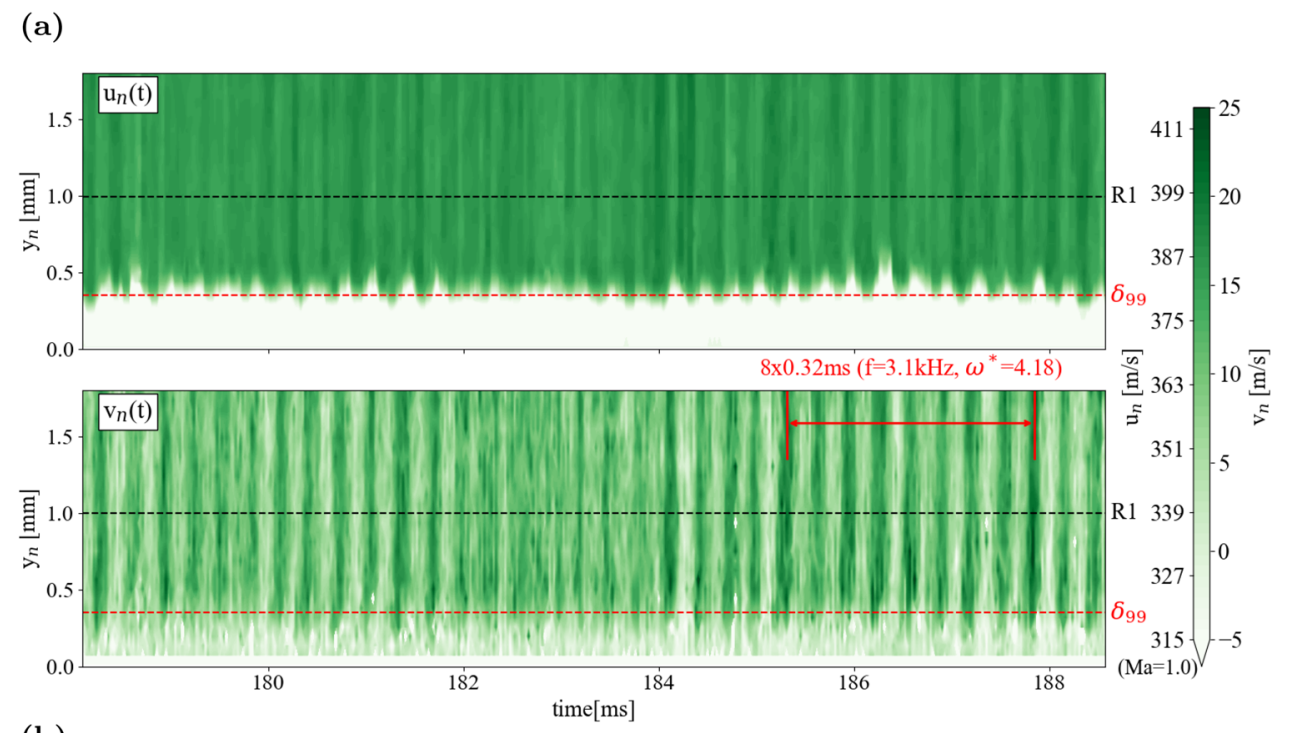

(b)
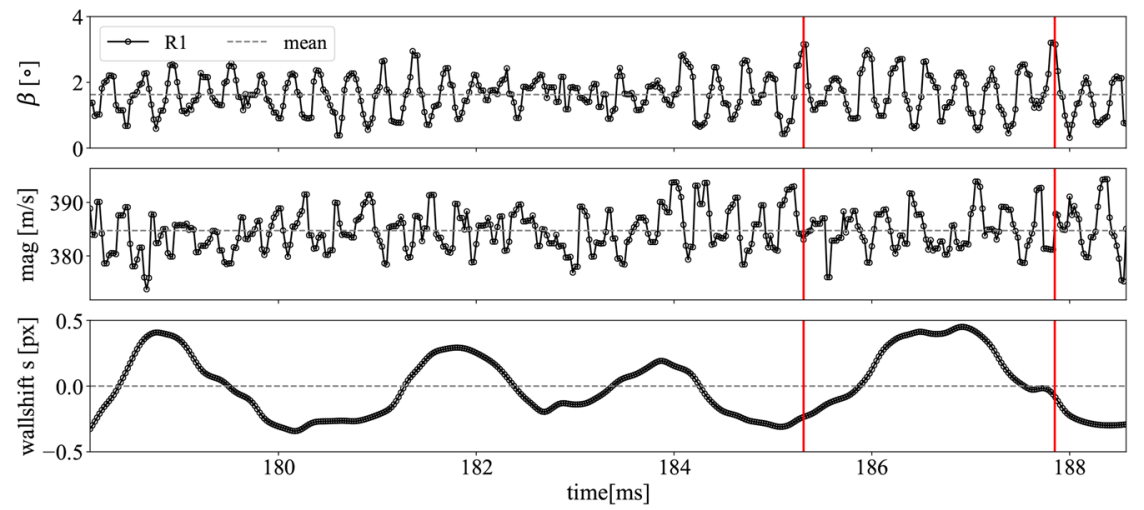

To further investigate the upstream signature of the buffeting quantitatively, the HS-PIV data of the inlet BL at the ODP were sampled along the streamlines $A, B$ and $C$ (cf. Fig. 11) which run along wall distances of $0.8 \delta_{0}, 2.5 \delta_{0}$ and $4.6 \delta_{0}$ at $x_{c} / c=0.31$.

Figure 14a shows the chordwise power spectral densities of velocities along each streamline, while Fig. 14b shows sections of the PSDs at $x=8 \mathrm{~mm}$ and $x=18 \mathrm{~mm}$ corresponding to positions that lay $19 \mathrm{~mm}$ and $9 \mathrm{~mm}$ upstream of $\overline{x_{s}}$. Here, the estimations made with Fig. 13 are confirmed: Pronounced spectral peaks are present at the dominant buffet frequencies 1.6 and $3.3 \mathrm{kHz}$ for the near-wall streamline $A$ both in the wall-parallel and in the transverse component. While for the transverse component the PSDs of dominant shock oscillations are approximately constant over the wall distance, they vanish outside of the BL for the longitudinal component $u_{n}$, because at supersonic speed longitudinal velocity variations cannot enter directly this region. The power density at these frequencies becomes significantly weaker from $x=13 \mathrm{~mm}$ (14 mm upstream from $\left.\overline{x_{s}}\right)$, indicating wave propagation toward upstream and away from the shock foot.
In addition, Fig. 14 indicates distinct harmonic low-frequency components at $370 \mathrm{~Hz}\left(\omega^{*}=0.49\right)$ for the transverse component $v_{n}$ which appear in the spectra over the complete chordwise extent. These disturbances hardly show up in the spectra of the shock oscillations (cf. Fig. 7b). Wall-shift spectra from blade displacements in the same ROI (Inflow $B L$ ) indicate a coincidence of a weak blade or camera oscillation mode at $370 \mathrm{~Hz}$ which biases the wall normal velocity measurement.

Following Hartmann et al. (2013), wave propagation velocities of shock-induced perturbations can be estimated from time-resolved PIV data through linear interpolation of local maxima of the correlation coefficients of two-point two-time velocity correlations. The cross-correlation coefficient of velocities at two positions $i, j$ along each streamline is defined as:

$R_{i, j}(\tau)=\frac{u_{i}(t) u_{j}(t+\tau)}{\sigma_{u_{i}} \sigma_{u_{j}}}$,

where $\tau$ is the temporal lag, $u$ is the wall-parallel velocity, and $\sigma$ denotes the velocity standard deviation at each 
Fig. 14 a Spectra of velocity fluctuations upstream of the shock-foot and along the streamlines $A, B, C$ as shown in Fig. 11 ; b spectra at two chordwise positions as indicated in (a); the bin spacing is $20 \mathrm{~Hz}$ (a)
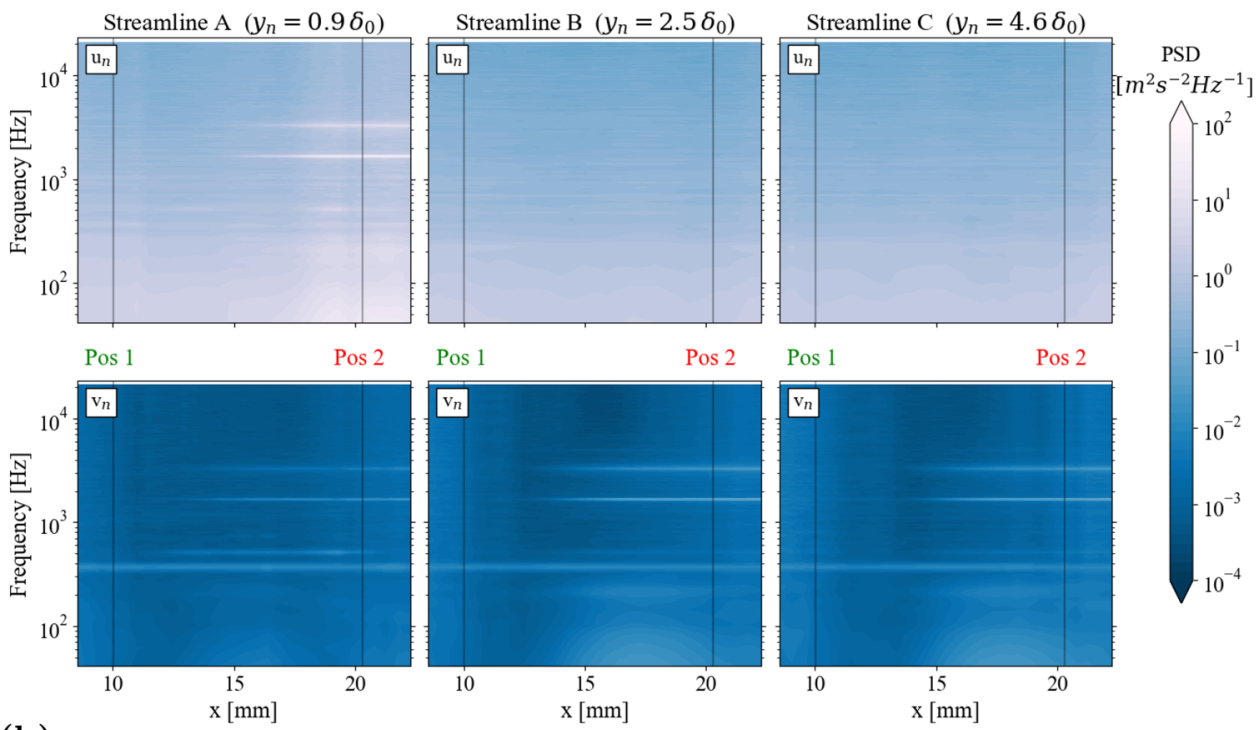

(b)
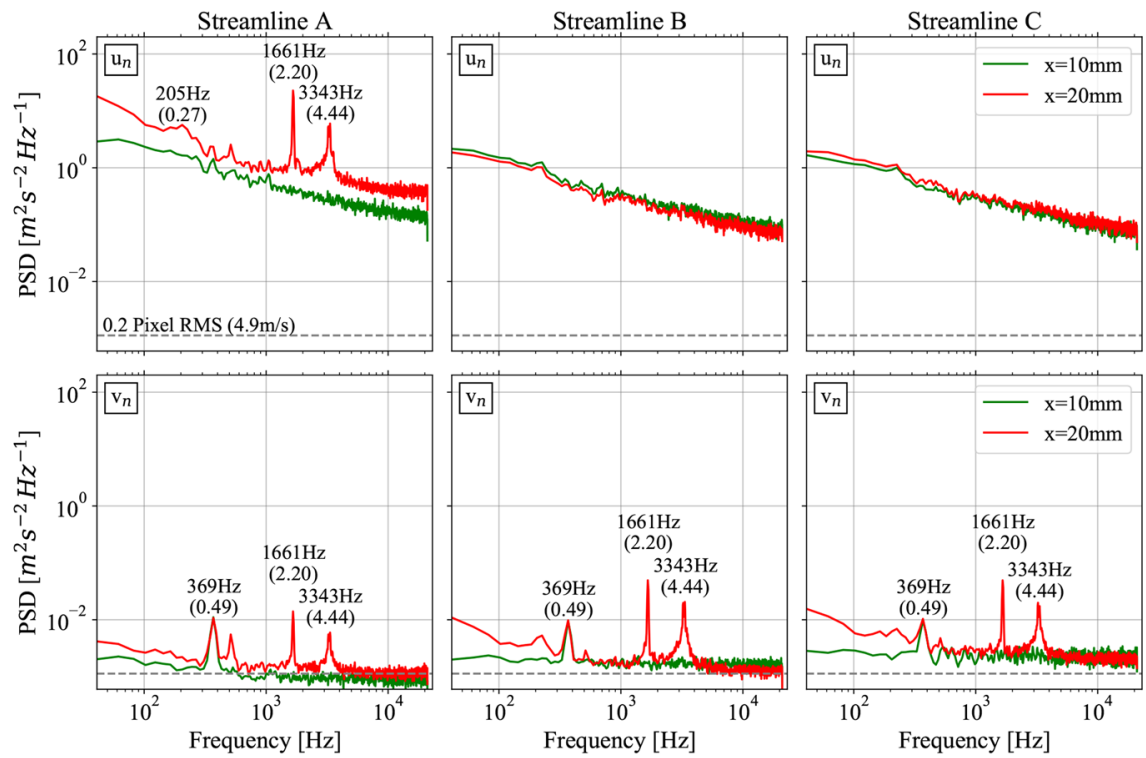

position. For evaluations of the inflowing BL, the reference position $i$ is located near the downstream edge of the measurement domain which is located $5.5 \mathrm{~mm}$ upstream of $\overline{x_{s}}$.

Figure 15 indicates a periodic modulation with time delays that correspond to the dominant shock buffet frequencies 1.7 and $3.2 \mathrm{kHz}$. Similar to evaluations by Hartmann et al. (2013), estimates of wave propagation velocities of these velocity perturbations are obtained from the slope of a linear fit of local correlation maxima along the chordwise coordinate. To reduce the fit residuals, the time traces were high-pass-filtered prior to cross-correlation using a 5th-order Butterworth filter with a cutoff frequency of $1.5 \mathrm{kHz}$. From Fig. 15, it is evident that shock-induced transverse perturbations propagate upstream approximately at $-52 \mathrm{~m} / \mathrm{s}$.
Shock-induced wall-parallel perturbations $\left(u_{i} * u_{j}\right)$ can only propagate upstream in the subsonic BL (streamline $A$ ).

\subsection{Boundary layer thickening downstream of the shock-foot}

Figure 16 displays sequential PIV samples of the buffet flow downstream of the shock foot recorded in the third measurement field (region $S B L I$ ) obtained at $35 \mathrm{kHz}$ sampling rate. Within these four samples, the shear layer downstream of the shock-foot exhibits high unsteadiness with strongly intermittent vortices, during which the shock foot only slightly moves. 
Fig. 15 Two-point correlations of velocities upstream of the shock foot along the streamlines $A, B, C$ in Fig. 11 and least square fit of linear relations between distance and time delay; Reference position: $-\cdot-$.
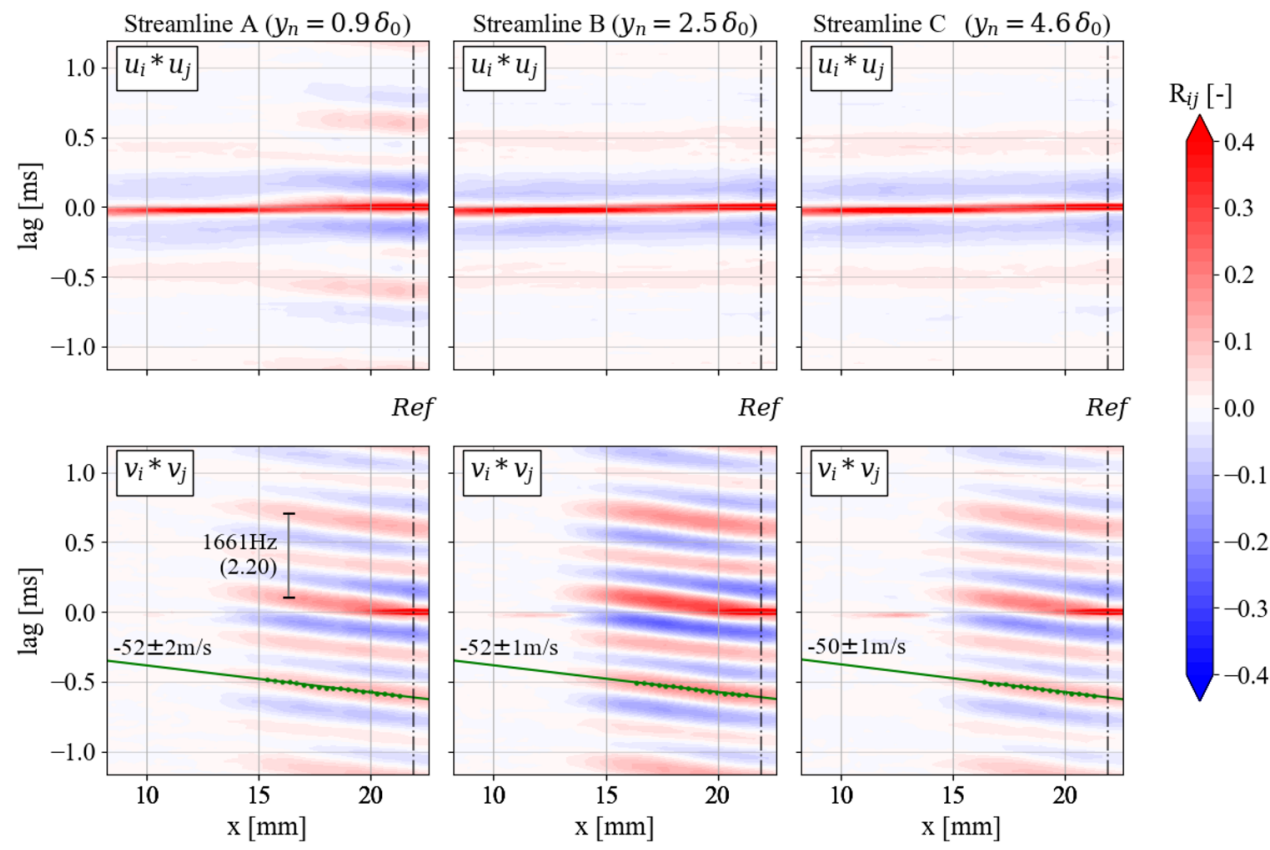

1

.2

3

0.4

gradient becomes visible and indicates the shock-induced BL thickening, whereby neither shock-induced bubbleseparation nor pronounced reverse flow becomes visible which indicates a weak interaction according to Clemens and Narayanaswamy (2014). Despite the presence of strong, self-sustained shock oscillations in the flow, only minor differences can be observed regarding the rate of change of velocity normal to the blade surface for the most upstream and downstream shock positions. At the most downstream shock position, the BL thickening is slightly less pronounced compared to the most upstream shock position.

For a supercritical airfoil under shock buffet, Hartmann et al. (2013) described that the variation of strength of the shock-induced vortical structures in the shear layer depends on the relative velocity of the shock front which is due to relatively different pre-shock Mach numbers. Although not so pronounced, this effect can also be observed for the present flow. Figure 17(right) shows the conditionally averaged axial velocities in a sample column $6 \mathrm{~mm}$ downstream of $\overline{x_{s}}$. Velocity profiles are conditionally sampled on the basis whether the shock wave was moving upstream $\left(u_{s} \leq 0\right)$ or downstream $\left(u_{s}>0\right)$ through each shock location. Due to the high wall-normal velocity gradient, the height of the PIV interrogation window is not small enough to achieve zero velocity on the wall and the measured velocity increases in the further course due to particle reflections on the wall. However, if the blade surface is assumed to coincide at the velocity minimum and robust velocity measures are accepted only from a height where the interrogation window no longer intersects with the wall (the curve starting from the symbol in Fig. 17(right)), it can be deduced that the velocity profile
To demonstrate the spatial evolution of the downstream flow with shock position, PIV data were conditionally averaged after the passage shock location from shadowgraphs at a resolution of $1 \mathrm{~mm}(1 / 70$ of $c)$. Figure 17 shows the corresponding velocities for $x_{s}= \pm 1.5 \mathrm{~mm}$ and for $\bar{x}_{s}$. The developing shear layer in the adverse pressure 
Fig. 17 Isentropic Mach numbers $\mathrm{Ma}_{\mathrm{is}}$ conditionally averaged after shock positions at a resolution of $1 \mathrm{~mm}(1 / 70$ of $c$ ) at $x_{s}-\overline{x_{s}}=-1.5 \mathrm{~mm}$ (top);0 mm (middle); $1.5 \mathrm{~mm}$ (bottom); On the right, the corresponding wall parallel velocity distributions are shown at the indicated sample column, averaged for all samples of the corresponding shock-position bin and for downstream $\left(u_{s}>0\right)$ and upstream $\left(u_{s} \leq 0\right)$ passages of the shock wave
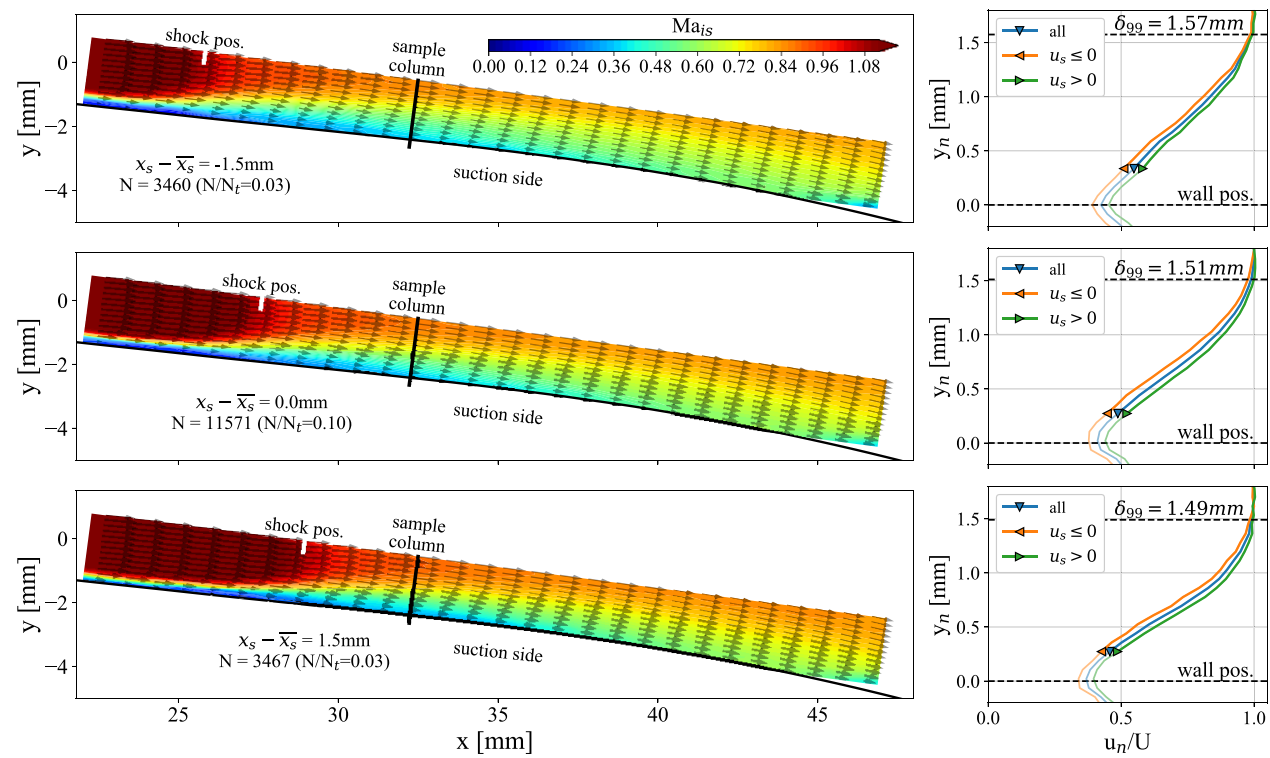

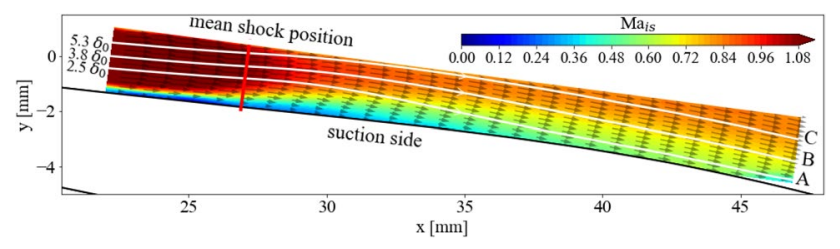

Fig. 18 Mean isentropic Mach numbers $\mathrm{Ma}_{\mathrm{is}}$ in the SBLI measurement region at the ODP and streamlines along which velocity spectra and two-time correlations were evaluated

becomes fuller when the shock front moves downstream $\left(u_{s}>0\right)$, indicating a higher momentum contained in the TBL.

\subsection{Propagation of periodic disturbances out of the SBLI region}

To evaluate the chordwise presence and strength of dominant buffet frequencies in the velocity spectra, Fourier analysis is conducted based on PIV timetraces extracted along the coordinates of the time-averaged streamlines $A, B$ and $C$ as shown in Fig. 18. The wall-normal distance of the entry points of each streamline is given in BL thicknesses $\delta_{0}$. These streamlines do not correspond to the velocity field in a single shot, but allow to follow velocity variations along the mean flow field in consideration of the blade curvature. Measured velocities at each streamline coordinate are split into the tangential and transverse components $u_{n}$ and $v_{n}$ to allow distinctions between fluctuations that are longitudinal and transverse to the mean flow.

Figure 19a compares the spectra along the time-averaged streamlines $A, B$ and $C$, and Fig. $19 \mathrm{~b}$ additionally shows the PSDs at the mean shock location and further downstream in an area where the outer flow approaches the suction side again. As expected, an extended low-frequency broadband content for $f<1 \mathrm{kHz}$ is visible in the region near the oscillating shock foot, which is also found in the shock motion spectrum (cf. Fig. 7b). This part is strongly attenuated further downstream and is hardly visible at $x=40 \mathrm{~mm}$. Less damped, dominant frequencies of the shock buffet at $\omega^{*}=2.28$ and 4.53 are propagated downstream, whereby oscillations of the longitudinal component $u_{n}$ experience additional damping with increasing distance to the blade surface. Considering the shock oscillation range of about $( \pm 3 \mathrm{~mm})$ around $\overline{x_{s}}$ one can see that upstream of the shock position only transverse velocity components $v_{n}$ oscillate at dominant buffet frequencies.

To get an improved insight into the dynamics between shock motion and velocity variations, the time trace of the shock position $x_{s}(t)$ was correlated with the velocity time traces $u_{n}(t)$ and $v_{n}(t)$ along the chordwise positions $j$, where the $x_{s}^{\prime} * u_{n}$ correlation is

$R_{s, j}(\tau)=\frac{x_{s}^{\prime}(t) u_{j}(t+\tau)}{\sigma_{x_{s}^{\prime}} \sigma_{u_{j}}}$,

where $x_{s}^{\prime}$ is the high-pass-filtered time trace of shock excursions. High pass filtering using a Butterworth filter at $f_{g}$ $=1.5 \mathrm{kHz}$ was applied to shock traces to suppress fractions of low-frequency shock motion, not associated with frequency-locked shock buffet at $\mathrm{f}=1.66$ and $3.3 \mathrm{kHz}$.

The $x_{s}^{\prime} * u$ correlation for streamlines $A$ and $B$ in Fig. 20 indicates that starting approximately from $x=34 \mathrm{~mm}$, disturbances travel with the longitudinal velocity variations in both directions, downstream at approximately $98 \mathrm{~m} / \mathrm{s}$ and upstream at $-78 \mathrm{~m} / \mathrm{s}$, but only up to the shock front plus its fluctuation range. It is assumed that these measures 
(a)
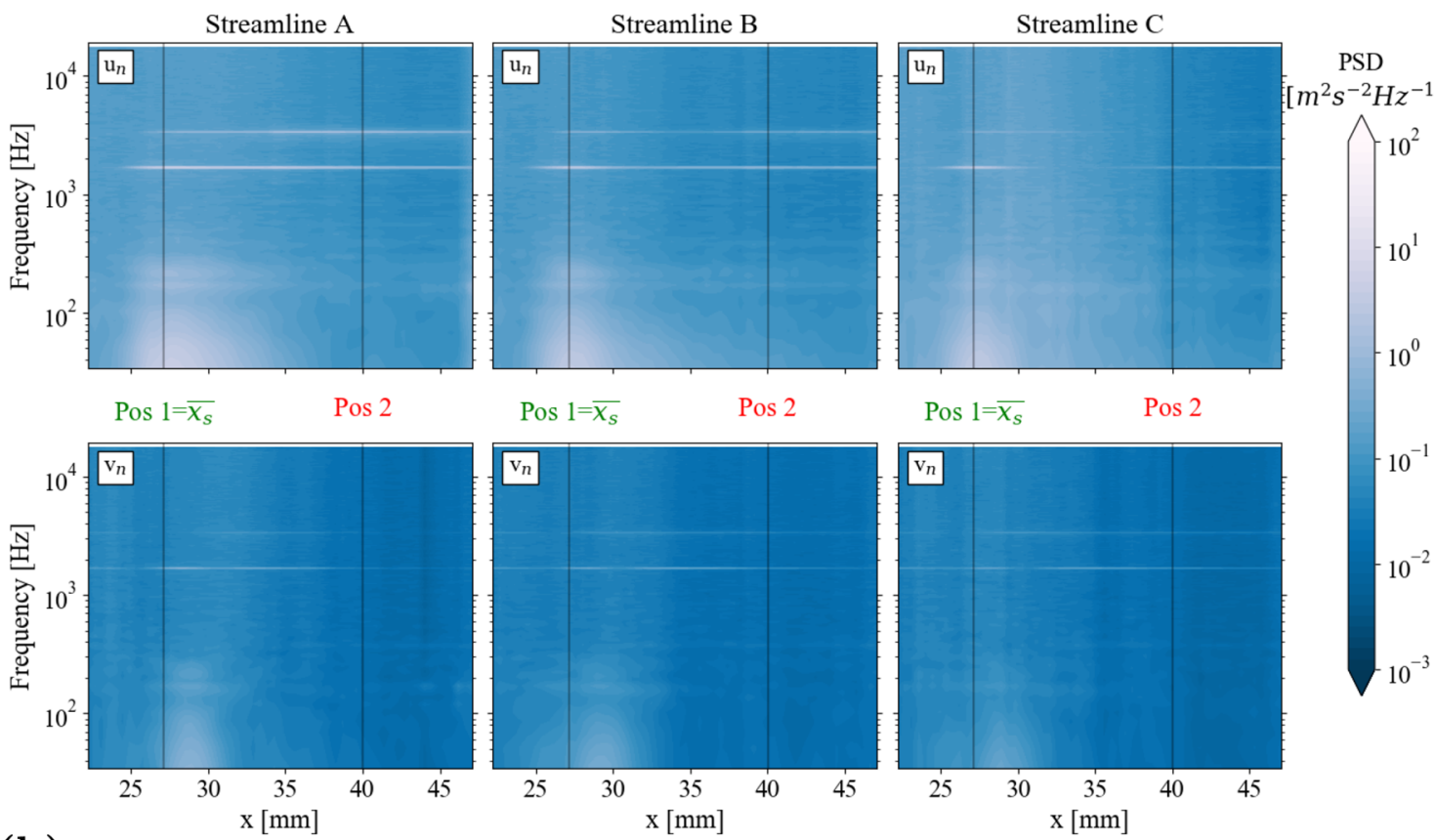

(b)
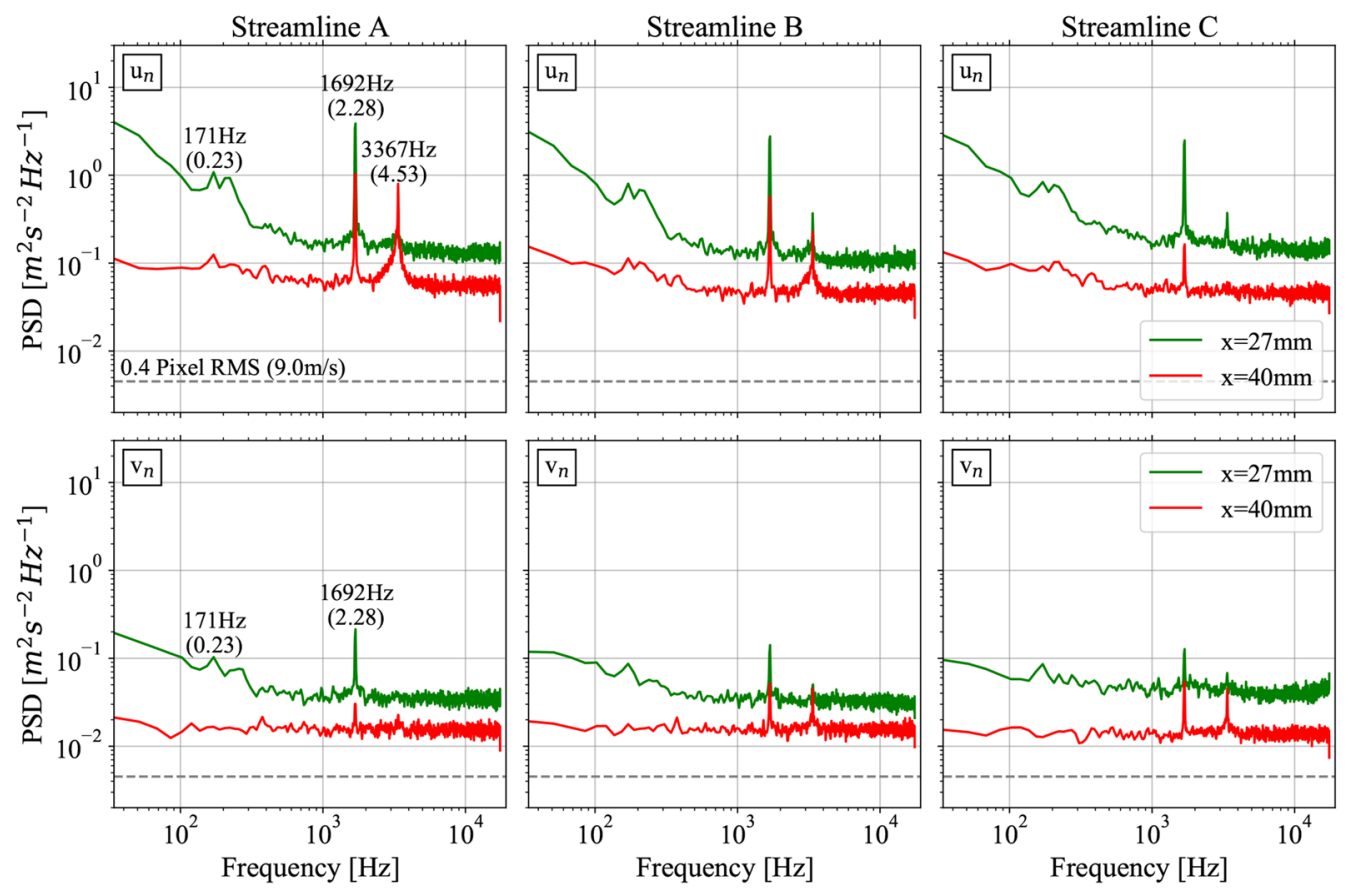

Fig. 19 Spatially resolved periodograms obtained in the SBLI region along streamlines $A, B, C$ in Fig. 18 at the ODP a chordwise spectra of longitudinal and transverse velocity fluctuations; $\mathbf{b}$ spectra at two positions marked by vertical lines in (a); the bin spacing is $17 \mathrm{~Hz}$

correspond to the propagation velocities at which longitudinal disturbances propagate in the velocity field in phase with the high-frequency part of the shock motion. Correlations of $x_{s}^{\prime} * u$ for streamline $C$ additionally exhibit a downstream propagation at $35 \mathrm{~m} / \mathrm{s}$ indicating the occurrence of a two-way propagation mechanism between $\overline{x_{s}}$ and $x=34 \mathrm{~mm}$.

The correlation $x_{s}^{\prime} * v_{j}$ (cf. Fig. 20) exhibits a phase change of $\pi$ across the shock with alternating correlation coefficients upstream and downstream of the shock. Similar 
Fig. 20 Correlation of highpass-filtered time traces of shock excursions and velocity in the SBLI region along the streamlines $A, B, C$ in Fig. 18 and least square fit of linear relations between distance and time delay

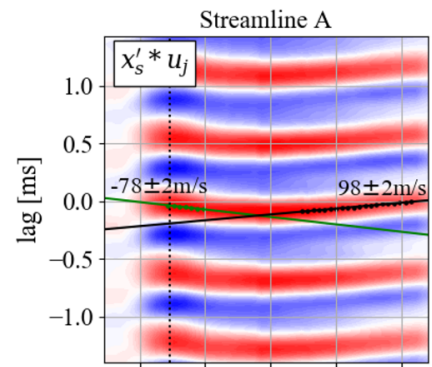

$\overline{\chi_{s}}$

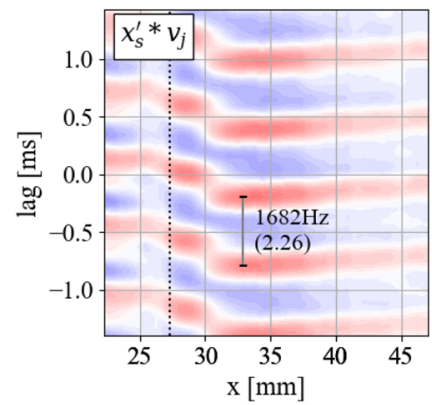

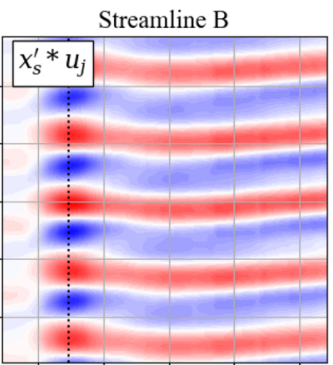

$\overline{\chi_{s}}$

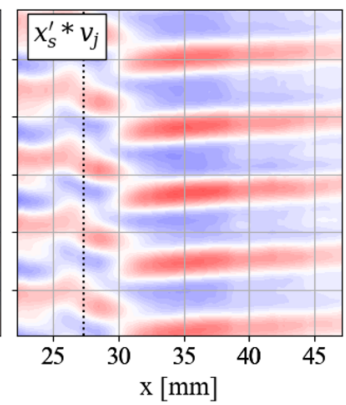

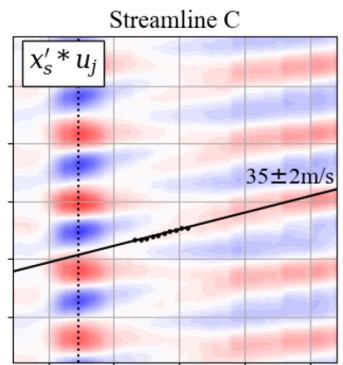

$\overline{\chi_{s}}$

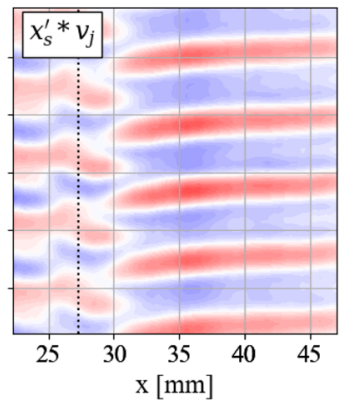

$\mathrm{R}_{s j}[-]$

$-0.20$

$-0.15$

$-0.10$

$-0.05$

$-0.00$

$-0.05$

$-0.10$

$-0.15$

$-0.20$

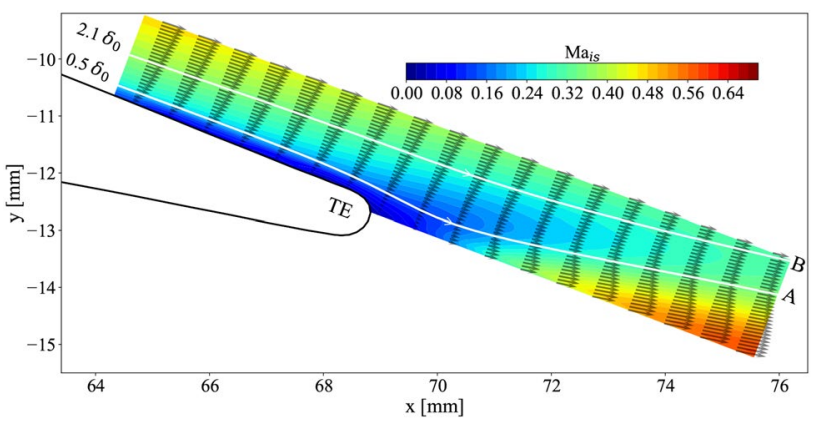

Fig. 21 Mean isentropic Mach numbers $\mathrm{Ma}_{\mathrm{is}}$ near and beyond the trailing edge at the ODP and streamlines along which velocity spectra and two-point correlations were evaluated phase shifts have also been reported from two-point correlations of time-resolved surface pressures across shocks in the buffet flow on a supercritical airfoil (Hartmann et al. 2013). This means that at the same time-lag with respect to $x_{s}(t)$ always alternating transverse velocities occur in front and below the shock $\left(v_{1}(t)>\bar{v}\right.$ if $v_{2}(t)<\bar{v}$ and vice versa).

\subsection{Propagation of periodic disturbances over the trailing edge}

As recapped in the introduction, self-sustaining shock oscillations on supercritical airfoils can be related to acoustic feedback which originates from shock-induced instability
Fig. 22 Spatially resolved periodograms obtained near the trailing edge along streamlines $A$ and $B$ in Fig. 21; a spectra of longitudinal and transverse velocity fluctuations along $x$; b spectra at two positions marked by vertical lines in (a); the bin spacing is $24 \mathrm{~Hz}$ (a)
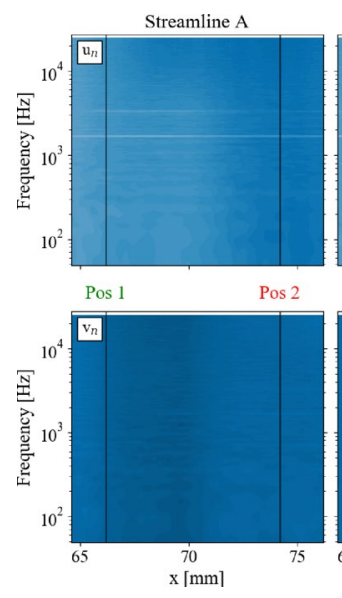

(b)
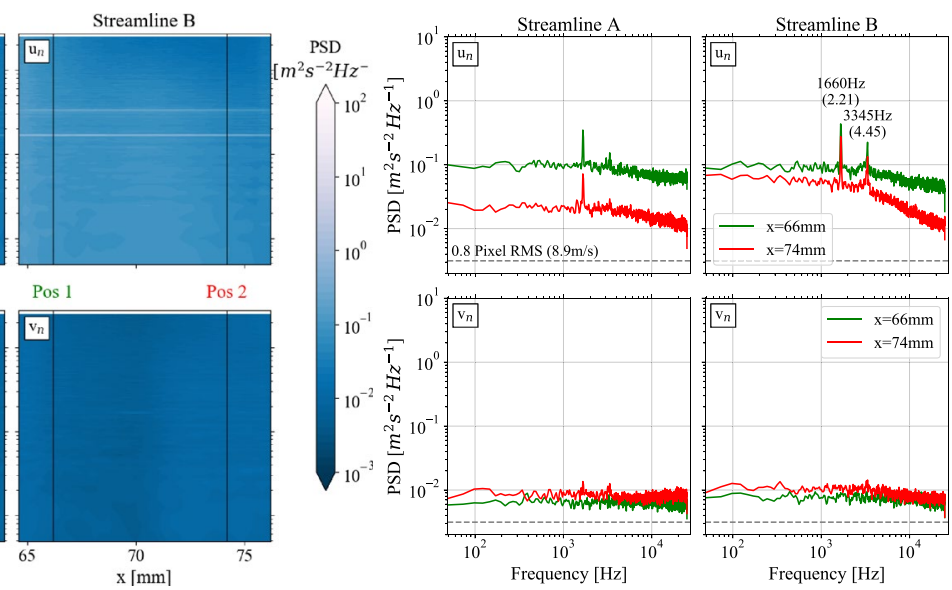
waves convecting over the TE (Lee 2001; Hartmann et al. 2013).

To determine whether instability waves are also convected toward the TE in the present buffeting flow, a Fourier analysis is conducted using PIV velocity time traces extracted along the coordinates of the time-averaged streamlines $A$ and $B$ shown in Fig. 21. Streamline $A$ partially passes through the shear layer on the pressure side flow in the wake of the blade.

Figure 22 indicates that along the time-averaged streamlines $A$ and $B$, dominant buffet frequencies at $f=1.66$ and $3.3 \mathrm{kHz}$ are present only in the longitudinal component $u_{n}$ and exhibit decreasing PSD with increasing downstream distance. Further excitations by wake interactions with the pressure side flow would also be conceivable, but this does not seem to be the case within the relevant frequency range. The two-point correlations $u_{i} * u_{j}$ shown in Fig. 23 also do not indicate any upstream convection of disturbances that could trigger the shock motion.

\section{Discussion}

For the buffeting flow, the PSDs of $x_{s}$ indicate tonal shock oscillations at $1.68 \mathrm{kHz}$ and $3.4 \mathrm{kHz}\left(\omega^{*}=2.3\right.$ and 4.5$)$ which are not caused by aeroelastic excitation from structural blade vibrations.

For these buffet frequencies, Lee's hypothesis could not be demonstrated for the present flow by means of an acoustic feedback loop that is established between $\overline{x_{s}}$ and the TE.

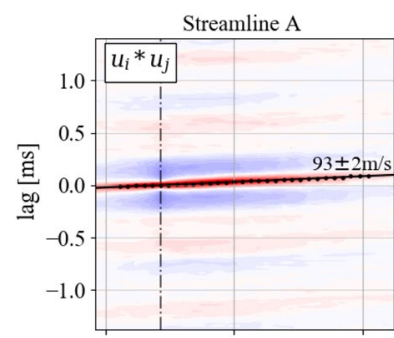

Ref
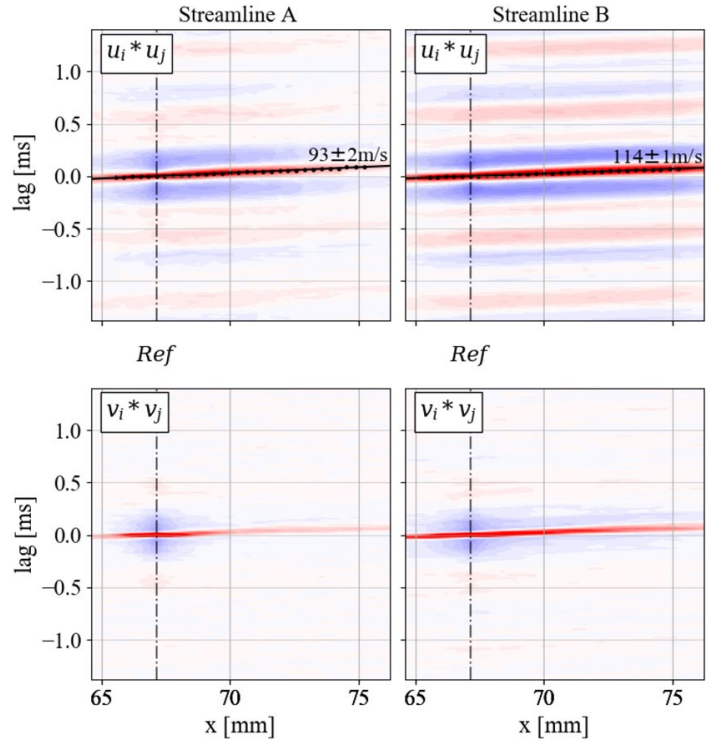

Ref

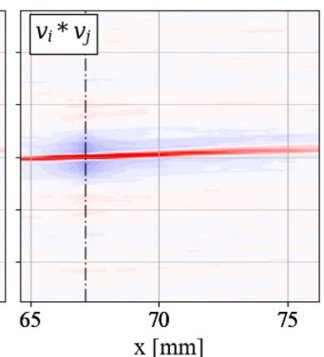

Fig. 23 High-pass-filtered two-point correlations of velocities near the TE along the streamlines $A$ and $B$ in Fig. 21 and least square fit of linear relations between the chordwise distance and time delay; reference position: $-\cdot-$.
This is reflected in the fact that two-point correlations did not show an upstream propagation of acoustic waves from the TE and thus the resonance conditions in Eqs. (1) and (2) could not be applied.

Also, at the dominant buffet frequencies no acoustic wave propagation, neither from the upstream bow shock nor from the leading edge flow, could be observed. This does not indicate a mechanism as proposed by Jacquin et al. (2009) and Garnier and Deck (2010) where phase-locked acoustic waves that originate from the TE travel upstream on the pressure side and move around the leading edge before interacting on the shock from upstream.

Rather, the results suggest that for the present flow shock buffet at $\omega^{*}=2.3$ and 4.5 is associated with shock-induced pressure fluctuations that originate from the low-momentum region immediately downstream of the shock foot near $x=34 \mathrm{~mm}$ and travel upstream (and downstream) in the form of longitudinal velocity oscillations. This is observable in Fig. 20 in a two-way propagation where for streamline $A$ periodic disturbances are propagated toward the shock through longitudinal velocities at $-78 \mathrm{~m} / \mathrm{s}$ and downstream in the upper part of the thickened BL through streamline $C$ at $35 \mathrm{~m} / \mathrm{s}$. While longitudinal velocity oscillations propagate upstream only through the subsonic low momentum region, transverse oscillations $x_{s}^{\prime} * v_{j}$ show a distinct correlation across the shock also outside the upstream BL. This correlation exhibits a phase shift of $\pi$, meaning that the temporal variation of transverse velocity with shock motion is in opposite phase upstream and downstream of the shock.

One possible explanation for such an interaction across the shock is that acoustic waves propagate upstream through the subsonic shear layer underneath the shock tip, driven by the pressure rise $p_{2} / p_{1}$ across the shock, which is 1.8 for the present conditions and which oscillates with the shock motion velocity approximately between 1.7 and 1.9 based on a peak-to-peak span of shock velocities of $\pm 11 \mathrm{~m} / \mathrm{s}$. Upstream of the shock, these waves can propagate inside the subsonic BL through the longitudinal and transverse velocity field (cf. Fig. 15) and can induce phase-locked variations of the flow angle over a wall-normal distance of at least $4.6 \delta_{0}$ (cf. Fig. 13b), which are assumed to influence the shock position. Upstream effects of pressure fluctuations through the subsonic shear layer below the shock tip have already been observed in DNS studies of shock-induced flow separations, where upstream propagation of acoustic waves affects the position of the separation point (cf. Pirozzoli and Grasso 2006).

Whether the different propagation velocities of the periodic disturbances upstream and downstream of the shock indicate a resonance through the subsonic shear layer underneath the shock foot was verified by relating the dominant buffet frequency $f=1 / T_{b}$ to the respective propagation velocities and characteristic lengths 
$T_{b}=\left(\frac{1}{a_{1 d}}+\frac{1}{\left|a_{1 u}\right|}\right)\left(\overline{x_{s}}-x_{1}\right)+\left(\frac{1}{a_{2 d}}+\frac{1}{\left|a_{2 u}\right|}\right)\left(x_{2}-\overline{x_{s}}\right)$,

where $x_{1}$ and $x_{2}$ are the most upstream and downstream positions between which the upstream propagation of disturbances occur, $\bar{x}_{s}$ is the mean shock location and $a_{1 u}, a_{1 d}$, $a_{2 u}, a_{2 d}$, respectively, denote the upstream and downstream wave propagation velocities on both sides of the shock. The first and seconds terms also correspond to the time required for disturbances to propagate from $x_{s}$ to $x_{1}$ and $x_{2}$, and back to $x_{s}$. It is assumed that the oscillating pressure equalization underneath the shock tip leads to a merging of both loops which corresponds to the dominant buffet frequency.

Based on Fig. $15 x_{1}=13.2 \mathrm{~mm}$ and $a_{1 u}=-52 \mathrm{~m} / \mathrm{s}$ and $a_{1 d}$ is estimated with the speed of sound $c_{1}=315 \mathrm{~m} / \mathrm{s}$. Downstream of the shock $x_{s}^{\prime} * u$ correlations revealed a two-way propagation in between $\overline{x_{s}}=27.3 \mathrm{~mm}$ and $x_{2}=34.0 \mathrm{~mm}$ with $a_{2 u}=-78 \mathrm{~m} / \mathrm{s}$ in the lower region of the BL (cf. streamline $A$ in Fig. 20) and with $a_{2 d}=35 \mathrm{~m} / \mathrm{s}$ in the upper region of the $\mathrm{BL}$ for streamline $C$. The corresponding estimate of the dominant buffet frequency with Eq. (7) yields $f=1.68 \mathrm{kHz}$ which coincides with the dominant peak at $f=1.683 \pm 0.006 \mathrm{kHz}$ in the PSD of $x_{s}(t)$ of Fig. 7b.

Here it should be noted that uncertainties of the propagation velocity $a_{1 u}$ and $a_{2 d}$ of $\pm 2 \mathrm{~m} / \mathrm{s}$ as in indicated in Fig. 20 account for a 2 percentage difference in the estimated buffet frequency.

\section{Conclusions}

The source of high-frequency self-sustained shock oscillations was investigated for a shock-BL interaction in the blade passage of a transonic compressor cascade model. The investigations are based on time-resolved PIV measurements at four stations in the cascade at sampling rates up to $50 \mathrm{kHz}$ using two continuously pulsed DPSS lasers along with synchronized high-speed shadowgraph imaging, allowing the determination of spectra and of two-time correlations between velocities and between shock location and each velocity component.

Velocity measurements on the suction side of the center blade near the shock foot indicate a weak shock-BL interaction, recognizable by the absence of bubble separation in the time-averaged flow. Time-resolved measurements at three chord-wise stations revealed different wave propagation velocities of the shock-induced perturbations upstream and downstream of the shock. For the relevant buffet frequencies, transverse velocity fluctuations exhibit a correlation across the shock including a phase shift of $\pi$. Any feedback mechanism between the TE and the shock-foot (Lee's model) was not evident for the present cascade flow. Rather it was shown that shock-induced disturbances propagate phaselocked with shock oscillations at $1.68 \mathrm{kHz}$ and $3.4 \mathrm{kHz}$ $\left(\omega^{*}=2.3\right.$ and 4.5$)$ from a region $7 \mathrm{~mm}$ downstream of the mean shock foot position to a region $13 \mathrm{~mm}$ in front of the shock. Based on these observations, it is hypothesized that feedback between both positions occurs through the subsonic region below the shock tip. The results further indicate a resonance between both chordwise positions around the shock foot in between which shock-induced perturbations propagate with the period of the dominant shock oscillation coinciding with the time required for a disturbance to propagate between these two positions.

The missing link is the wave propagation of disturbances in front of the shock in the downstream direction, whose propagation velocity was assumed to be at the speed of sound and which probably either occurs within the lower $\mathrm{BL}$ or outside the region illuminated by the light-sheet, i.e., was not observable with PIV.

Furthermore, it was shown that transverse velocity oscillations in front of the shock occur at the first harmonic of the shock buffet and lead to phase-locked variations of the flow angle over a wall-normal distance of at least $4.6 \delta_{0}$ where it is not clear whether these variations cause the shock movement.

The interdependence of the mechanisms of shock-boundary layer interactions could be further clarified using spatially and temporally resolved surface-pressure measurements. In this regard, fast-response pressure sensitive paints (iPSP) have undergone considerable progress in recent years (Gößling et al. 2020; Hilfer et al. 2020) and quite possibly could offer insight to the propagation of pressure fluctuations on the blade surface that currently cannot be deduced from the time-resolved flow field data.

Acknowledgements We thank Lufthansa Technik AG for the very excellent cooperation.

Funding Open Access funding enabled and organized by Projekt DEAL.

Open Access This article is licensed under a Creative Commons Attribution 4.0 International License, which permits use, sharing, adaptation, distribution and reproduction in any medium or format, as long as you give appropriate credit to the original author(s) and the source, provide a link to the Creative Commons licence, and indicate if changes were made. The images or other third party material in this article are included in the article's Creative Commons licence, unless indicated otherwise in a credit line to the material. If material is not included in the article's Creative Commons licence and your intended use is not permitted by statutory regulation or exceeds the permitted use, you will need to obtain permission directly from the copyright holder. To view a copy of this licence, visit http://creativecommons.org/licenses/by/4.0/. 


\section{References}

Beresh S, Kearney S, Wagner J, Guildenbecher D, Henfling J, Spillers R, Pruett B, Jiang N, Slipchenko M, Mance J, Roy S (2015) Pulse-burst PIV in a high-speed wind tunnel. Meas Sci Technol 26(9):095305. https://doi.org/10.1088/0957-0233/26/9/095305

Beresh SJ, Henfling JF, Spillers RW, Spitzer SM (2018) "Postagestamp PIV": small velocity fields at $400 \mathrm{kHz}$ for turbulence spectra measurements. Meas Sci Technol 29(3):034011. https://doi. org/10.1088/1361-6501/aa9f79

Brown ML, Parsheh M, Aidun CK (2006) Turbulent flow in a converging channel: effect of contraction and return to isotropy. J Fluid Mech 560:437-448. https://doi.org/10.1017/S0022112006000449

Clemens NT, Narayanaswamy V (2014) Low-frequency unsteadiness of shock wave/turbulent boundary layer interactions. Annu Rev Fluid Mech 46(1):469-492. https://doi.org/10.1146/annurev-fluid $-010313-141346$

Crouch JD, Garbaruk A, Magidov D, Travin A (2009) Origin of transonic buffet on aerofoils. J Fluid Mech 628:357-369. https://doi. org/10.1017/S0022112009006673

Garnier E, Deck S (2010) Large-eddy simulation of transonic buffet over a supercritical airfoil. In: Deville M, Lê TH, Sagaut P (eds.) Turbulence and Interactions, pp. 135-141. Springer Berlin Heidelberg, Berlin, Heidelberg (2010). https://doi.org/10.1007/9783-642-14139-3 16

Giannelis NF, Vio GA, Levinski O (2017) A review of recent developments in the understanding of transonic shock buffet. Progress in Aerospace Sciences 92:39-84 https://doi.org/10.1016/j.paero sci.2017.05.004.http://www.sciencedirect.com/science/article/pii/ S0376042117300271

Gößling J, Ahlefeldt T, Hilfer M (2020) Experimental validation of unsteady pressure-sensitive paint for acoustic applications. Exp Thermal Fluid Sci 112:109915. https://doi.org/10.1016/j.expth ermflusci.2019.109915

Hartmann A, Feldhusen A, Schröder W (2013) On the interaction of shock waves and sound waves in transonic buffet flow. Phys Fluids 25(2):026101. https://doi.org/10.1063/1.4791603

Hergt A, Grund S, Steinert W (2016) Webpage of the transonic cascade wind tunnel. http://www.dlr.de/at/en/desktopdefault.aspx/tabid -1549/2461_read-3834/

Hergt A, Klinner J, Wellner J, Willert C, Grund S, Steinert W, Beversdorff M (2019) The Present Challenge of Transonic Compressor Blade Design. J Turbomach 141(9) https://doi. org/10.1115/1.4043329

Hilfer M, Klein C, Mayer F (2020) Aeroacoustic measurements with fast response pressure sensitive paint in a cavity at transonic mach numbers. In: Deutscher Luft- und Raumfahrtkongress 2020 (virtuell). https://elib.dlr.de/136605/

Jacquin L, Molton P, Deck S, Maury B, Soulevant D (2009) Experimental study of shock oscillation over a transonic supercritical profile. AIAA J 47(9):1985-1994. https://doi.org/10.2514/1.30190

Klinner J, Hergt A, Grund S, Willert CE (2019) Experimental investigation of shock-induced separation and flow control in a transonic compressor cascade. Exp Fluids 60(6):96. https://doi.org/10.1007/ s00348-019-2736-z

Klinner J, Hergt A, Willert C (2014) Experimental investigation of the transonic flow around the leading edge of an eroded fan airfoil. Exp Fluids 55(9):1800. https://doi.org/10.1007/s0034 8-014-1800-y

Lee B (2001) Self-sustained shock oscillations on airfoils at transonic speeds. Prog Aerosp Sci 37(2):147-196. https://doi.org/10.1016/ S0376-0421(01)00003-3

Lee BHK (1990) Oscillatory shock motion caused by transonic shock boundary-layer interaction. AIAA J 28(5):942-944. https://doi. org/10.2514/3.25144

Michaelis D, Neal DR, Wieneke B (2016) Peak-locking reduction for particle image velocimetry. Meas Sci Technol 27(10):104005. https://doi.org/10.1088/0957-0233/27/10/104005

Miller JD, Jiang N, Slipchenko MN, Mance JG, Meyer TR, Roy S, Gord JR (2016) Spatiotemporal analysis of turbulent jets enabled by $100-\mathrm{kHz}, 100-\mathrm{ms}$ burst-mode particle image velocimetry. Exp Fluids 57(12):192. https://doi.org/10.1007/s00348-016-2279-5

Pirozzoli S, Grasso F (2006) Direct numerical simulation of impinging shock wave/turbulent boundary layer interaction at $\mathrm{M}=2.25$. Phys Fluids 18(6):065113. https://doi.org/10.1063/1.2216989

Scharnowski S, Bross M, Kähler CJ (2018) Accurate turbulence level estimations using PIV/PTV. Exp Fluids 60(1):1. https://doi. org/10.1007/s00348-018-2646-5

Schlichting H, Gersten K (2017) Boundary-Layer Theory. Springer Berlin Heidelberg, Berlin, Heidelberg. https://doi.org/10.1007/9783-662-52919-5

Westerweel J, Scarano F (2005) Universal outlier detection for PIV data. Exp Fluids 39(6):1096-1100. https://doi.org/10.1007/s0034 8-005-0016-6

Willert C, Stasicki B, Klinner J, Moessner S (2010) Pulsed operation of high-power light emitting diodes for imaging flow velocimetry. Measurement Science and Technology 21(7): 1-12. http://elib. dlr.de/64324/

Publisher's Note Springer Nature remains neutral with regard to jurisdictional claims in published maps and institutional affiliations. 